\title{
Paracrine role for endothelial IGF-1 receptor in white adipocyte beiging
}

Natalie J Haywood ${ }^{1}$, Katherine I Bridge ${ }^{\# 1}$, Cheukyau Luk ${ }^{\# 1}$, Nele Warmke ${ }^{1,3}$, Katie J Simmons ${ }^{1}$, Michael Drozd ${ }^{1}$, Amy Moran ${ }^{1}$, Sam Straw ${ }^{1}$, Jason L Scragg ${ }^{1}$, Jessica Smith ${ }^{1}$, Sunti Limumpornpetch ${ }^{1}$, Claire H Ozber ${ }^{1}$, Chloe G Wilkinson ${ }^{1}$, Anna Skromna ${ }^{1}$, Natallia Makava ${ }^{1}$, Andrew Walker ${ }^{1}$, Nicole T Watt ${ }^{1}$, Romana Mughal ${ }^{1,4}$, Kathryn J Griffin ${ }^{1}$, Hema Viswambharan ${ }^{1}$, Nadira Y Yuldasheva ${ }^{1}$, David J Beech ${ }^{1}$, Piruthivi Sukumar ${ }^{1}$, Antonio VidalPuig $^{2}$, Klaus K Witte ${ }^{1,5}$, Stephen B Wheatcroft ${ }^{1}$, Richard M Cubbon ${ }^{1}$, Lee D Roberts ${ }^{1}$, Mark T Kearney*1.

1. Leeds Institute of Cardiovascular and Metabolic Medicine, Faculty of Medicine and Health, University of Leeds, UK.

2. University of Cambridge Metabolic Research Laboratories, Cambridge, UK.

3. Current address - Integrative Vascular Biology Laboratory, Max Delbrück Center for Molecular Medicine in the Helmholtz Association, Berlin, Germany.

4. Current address - Department of Optometry and Vision Sciences, University of Huddersfield, UK.

5. Current address - Dept of Internal Medicine I, University Clinic, RWTH Aachen University, Aachen, Germany.

\# These authors contributed equally

*Corresponding author: m.t.kearney@leeds.ac.uk

\section{Acknowledgements}

We would like to acknowledge the histology service from the Division of Pathology and Data Analytics, Colorectal Pathology Trials, University of Leeds, for sectioning and staining adipose and liver samples. The Faculty of Biological Sciences Bioimaging Facility has received equipment grants from the Wellcome Trust to purchase confocal microscopes used in this project. MTK is the guarantor of this work and, as such, had full access to all the data in the study and takes responsibility for the integrity of the data and the accuracy of the data analysis.

NJH was funded by a British Heart Foundation Project Grant (PG/18/82/34120). CL was funded by a British Heart Foundation PhD studentship (FS/19/59/34896). MD was funded by a British Heart Foundation Clinical Research Training fellowship (FS/18/44/33792). NTW was funded by a British Heart Foundation Project Grant (PG/14/54/30939). LDR was funded by a Diabetes UK RD Lawrence Fellowship (16/0005382) and the Biotechnology and Biological Sciences Research Council (BB/R013500/1). SS is funded by a British Heart Foundation 
Clinical Research Training Fellowship (FS/CRTF/20/24071). CHO and RMC were funded by British Heart Foundation Clinical Intermediate Fellowships (FS/12/80/29821). AS was funded by a British Heart foundation Programme grant (RG/15/7/31521). MTK holds a British Heart Foundation Chair in Cardiovascular and Diabetes research, which also funded NM and KJS $(\mathrm{CH} / 13 / 1 / 30086)$.

Author contribution

$\mathrm{NJH}, \mathrm{KIB}, \mathrm{AS}, \mathrm{NM}$ and NYY performed in vivo experiments. NJH, KIB, NW, TS, AV and CHO performed ex vivo experiments. NJH, KIB, CL, KJS, AM, CHW and NTW performed cell culture and in vitro experiments. LDR performed metabolomic analysis. SS, JLS, JS, SL and KW obtained patient samples. $\mathrm{NJH}, \mathrm{KIB}, \mathrm{CL}, \mathrm{TS}, \mathrm{AV}, \mathrm{CHO}$ and $\mathrm{KG}$ performed image and data analysis. NJH, MD and MTK wrote the manuscript. DJB, LDR and RMC reviewed the manuscript. DJB, PS, KKW, SBW, RMC and MTK obtained funding.

\section{Declaration of Interests}

The authors declare no competing interests.

\section{Summary}

There are at least two distinct types of thermogenic adipocyte in mammals: a pre-existing form established during development, termed classical brown adipocytes and an inducible form, 'beige' adipocytes ${ }^{1-3}$. Various environmental cues can stimulate a process frequently referred to as 'beiging' of white adipose tissue (WAT), leading to enhanced thermogenesis and obesity resistance ${ }^{4,5}$. Whilst beiging of WAT as a therapeutic goal for obesity and obesity-related complications has attracted much attention ${ }^{6-9}$; therapeutics stimulating beiging without deleterious side-effects remain elusive ${ }^{10}$. The endothelium lines all blood vessels and is therefore in close proximity to all cells. Many studies support the possibility that the endothelium acts as a paracrine organ ${ }^{11-14}$. We explored the potential role of endothelial insulin-like growth factor-1 receptor (IGF-1R) as a paracrine modulator of WAT phenotype. Here we show that a reduction in endothelial IGF-1R expression in the presence of nutrient excess leads to white adipocyte beiging, increases whole-body energy expenditure and enhances insulin sensitivity via a non-cell autonomous paracrine mechanism. We demonstrate that this is mediated by endothelial release of malonic acid, which we show, using prodrug analogues, has potentially therapeutically-relevant properties in the treatment of metabolic disease.

Keywords: Endothelial cell, adipose tissue, IGF-1R, obesity, malonic acid, metabolomics. 
Over the past four decades, changes in human lifestyle have contributed to a pandemic of nutritional obesity ${ }^{15}$. In simple terms, obesity occurs due to sustained elevation of calorie intake, most often in the form of lipid and carbohydrate, and/or a decline in energy expenditure ${ }^{16}$. Disruption of this 'energy balance equation'17 can occur at any point in the human life course. In 2015, over 100 million children and 600 million adults were obese worldwide $^{18}$. An unfavourable deviation in the energy balance equation in favour of calorie excess results in ectopic deposition of lipids in tissues such as the liver and skeletal muscle, which are ill-equipped to deal with this challenge. As a result, deleterious perturbations in cellular function lead to type 2 diabetes mellitus, accelerated cardiovascular disease, fatty liver and some cancers ( ${ }^{19}$ for review).

Dietary lipids are stored in adipose tissue (AT) of which broadly speaking, there are two types. White AT (WAT) specialised for the storage of energy in the form of triglyceride during nutrient excess undergoes expansive remodelling with adipocytes adopting a hypertrophic/hyperplastic phenotype ${ }^{20}$. The second form of AT is brown AT (BAT) ${ }^{21}$. BAT, unlike WAT, expresses the mitochondrial carrier protein uncoupling protein-1 (UCP-1). UCP1 uncouples cellular respiration from mitochondrial ATP synthesis, affording BAT the capacity to oxidise lipids and glucose to generate heat ${ }^{21}$.

Recent studies indicate that at least two distinct types of thermogenic adipocyte exist in mammals: a pre-existing form established during development, termed 'classical brown', and an inducible form described as 'beige' ${ }^{4,5}$. BAT depots, previously thought to be limited to neonates, have also been identified in human adults ${ }^{1,2,3}$. Beige adipocyte biogenesis can be stimulated by various environmental cues, such as chronic cold exposure, ${ }^{4,5}$ in a process frequently referred to as 'beiging' of WAT. The potentially favourable metabolic effects of inducing more thermogenic AT in response to nutrient excess, has led investigators to seek new approaches to stimulate WAT beiging.

102 The insulin/insulin-like growth factor-1 (IGF-1) signalling system evolved millions of years ago

103 to co-ordinate organismal growth and metabolism. During evolution the insulin receptor (IR) 104 and IGF-1 receptor (IGF-1R) diverged from a single receptor in invertebrates, into a more 105 complex system in mammals consisting of the IR, IGF-1R and their respective ligands; insulin, 106 IGF-1 and IGF-II (22 for our review). We have previously shown that during calorie excess 107 circulating IGF-1 increases and IGF-1R levels decline, in a range of tissues including the vasculature ${ }^{23,24}$. 
109 The endothelium lines all blood vessels, and emerging data support the possibility that the

110 endothelium acts as a paracrine organ ${ }^{11-14}$, including, endothelial to AT signalling ${ }^{25-27}$.

111 Therefore, we explored the role of endothelial IGF-1R as a paracrine modulator in the

112 pathophysiology of obesity, and identified a novel, small molecule mediated beiging

113 mechanism.

115 Results

116 Murine endothelial IGF-1R knockdown enhances whole-body insulin sensitivity during positive 117 energy balance

118 To investigate the role of endothelial IGF-1R in the setting of increased energy balance, we 119 generated a tamoxifen-inducible, endothelial cell-specific IGF-1R knockdown mouse (ECIGF$1201 \mathrm{R}^{\mathrm{KD}}$ ) (Figure $1 \mathrm{~A}-\mathrm{B}$ ) with an $\mathrm{mTmG}$ reporter to confirm spatially appropriate Cre-recombinase 121 activity (Supplementary figure $1 \mathrm{~A}-\mathrm{C}$ ). When unchallenged on a standard laboratory chow diet 122 or challenged with high fat diet (HFD) for two weeks, ECIGF-1 $\mathrm{R}^{\mathrm{KD}}$ mice exhibited no difference 123 in body (Figure 1C) or organ weight (Figure 1D). Glucose tolerance was also unchanged in 124 both chow and HFD fed mice (Figure 1E-F). Insulin sensitivity was similar in chow fed-mice 125 (Figure 1G), but was enhanced in ECIGF-1 $\mathrm{R}^{\mathrm{KD}}$ after HFD feeding for two weeks, compared to 126 wildtype littermates on the same diet (Figure $1 \mathrm{H} \& \mathrm{I}$ ). After two weeks HFD, ECIGF-1R ${ }^{\mathrm{KD}}$ mice 127 had similar core body temperature (Supplementary Figure 2A) and fasting plasma 128 concentrations of glucose, insulin, IGF-I, free fatty acids, triglycerides, and leptin as wildtype 129 littermates on the same diet (Supplementary figure 2B-G).

131 Endothelial IGF-1R knockdown prevents deleterious remodelling of adipose tissue in the setting of increased energy balance

133 Historically, WAT was thought to be a simple storage depot for lipid. However, over the past 134 two decades, research has revealed WAT to be a complex and plastic organ (Reviewed ${ }^{28}$ ). 135 Targeting AT phenotype to mitigate against the adverse sequelae of obesity has thus received 136 significant attention (Reviewed ${ }^{29}$ ). Mechanisms of changing WAT from a storage to 137 thermogenic phenotype has been of particular interest ${ }^{6-9}$.

139 After two weeks of HFD, ECIGF-1 $R^{K D}$ mice displayed increased energy expenditure in relation 140 to body mass (Figure $2 \mathrm{~A}$ ), with no change in food intake or physical activity (Supplementary 141 figure 3A-F) compared to wildtype littermates. Epididymal WAT from ECIGF-1 $\mathrm{R}^{\mathrm{KD}}$ mice had 142 smaller adipocytes (Figure 2B-D), increased vascularity (Figure 2E\&F) and enhanced ex vivo 143 sprouting angiogenesis (Figure $2 \mathrm{G} \& \mathrm{H}$, Supplementary Figure $4 \mathrm{~A}$ ). This remodelling is 144 contrary to the deleterious phenotype in humans with either a high body mass index or raised $145 \mathrm{HbA1c}$ (Supplementary figure 5A-I). ECIGF-1R $\mathrm{R}^{\mathrm{KD}}$ mice had similar levels of epididymal WAT 
146 fibrosis, lipid accumulation in the liver and interscapular BAT (Supplementary figure 6A-F).

147 Vascularity in other AT depots (BAT, subcutaneous WAT and perinephric WAT) was

148 unchanged (Supplementary figure 6G-L), as well as vascularity of other organs including liver

149 and muscle (Supplementary figure 6M-P), suggesting an epididymal WAT specific effect of

150 reduced endothelial IGF-1R. Chow fed ECIGF-1 $R^{\mathrm{KD}}$ mice had similar AT vascularity

151 (Supplementary figure 7A\&B), indicative of a specific response to over nutrition. When fed

152 HFD for 2-weeks, ECIGF-1R ${ }^{\mathrm{KD}}$ also exhibited higher circulating levels of the beneficial

153 adipokine adiponectin (Figure 2I), an endogenous insulin sensitizer. Epididymal WAT from

154 HFD-fed ECIGF-1R $\mathrm{R}^{\mathrm{KD}}$ also exhibited a change in gene expression including upregulation of

155 Ucp-1, Vegfa and Cited1 (Figure 2J), indicative of adipocyte beiging, which was not seen in

156 chow-fed mice (Supplementary figure 7C).

158 As discussed above, at least two types of thermogenic adipocyte exist in mammals, BAT and

159 inducible 'beige' $\mathrm{AT}^{1-3}$. Beige adipocyte biogenesis may be stimulated by various

160 environmental cues ${ }^{4,5}$, a process referred to as 'beiging'. Here we show that a reduction in

161 endothelial IGF-1R expression in the presence of nutrient excess increases beige AT, increases whole-body energy expenditure, and enhances insulin sensitivity.

To explore the chronicity of these findings, a separate cohort of ECIGF-1 $\mathrm{R}^{\mathrm{KD}}$ mice and wildtype littermate controls received HFD for eight weeks. ECIGF-1 $R^{K D}$ mice maintained endothelial IGF-1R knockdown (Supplementary figure 8A\&B); body weight, organ weight, core body temperature and glucose tolerance were similar to wildtype littermate controls (Supplementary figure $8 \mathrm{C}-\mathrm{H}$ ). The enhanced insulin sensitivity of ECIGF-1 $\mathrm{R}^{\mathrm{KD}}$ seen at 2 weeks HFD persisted after 8 weeks of HFD (Supplementary figure 8I-J). Circulating insulin and IGF-1 concentrations were unchanged (Supplementary figure $8 \mathrm{~K}-\mathrm{L}$ ). Epididymal WAT from ECIGF-1 $\mathrm{R}^{\mathrm{KD}}$ mice after eight weeks HFD still had smaller adipocytes (Supplementary figure 9A\&B). ECIGF-1R ${ }^{K D}$ mice also had reduced lipid accumulation in BAT (Supplementary figure 9C\&D). There was no longer a difference in WAT vascularity (Supplementary figure 9E\&F); however, increased Vegf and Ucp-1 gene expression was retained (Supplementary figure 9G). There was no difference in WAT collagen deposition (Supplementary figure $9 \mathrm{H}-\mathrm{I}$ ) or fatty liver in ECIGF-1 $\mathrm{R}^{\mathrm{KD}}$ mice after 8 weeks HFD compared to wildtype littermate controls (Supplementary figure 9J\&K). Taken together, these findings suggest that the advantageous effects of EC IGF-1R knockdown on insulin sensitivity and AT phenotype are retained over longer periods of HFD.

181 To probe mechanisms underpinning the favourable changes to WAT in ECIGF-1 ${ }^{\mathrm{KD}}$ mice 182 receiving HFD, we investigated the possibility that adipocytes were directly derived from 
183 ECIGF-1R $\mathrm{R}^{\mathrm{KD}}$ endothelial cells, as it has been demonstrated that adipocytes of endothelial 184 origin exist in BAT and WAT ${ }^{30}$. However, in our model, Cre activity in ECIGF-1 $\mathrm{R}^{\mathrm{KD}}$ resulted in 185 vascular GFP expression, as intended, but no GFP expressing adipocyte like structures were 186 observed after 2 weeks of feeding, suggesting EC to AT transformation was not occurring 187 (Supplementary Figure 10A).

189 Since studies continue to emerge supporting the endothelium as a paracrine organ ${ }^{11-14}$, we 190 investigated a potential paracrine mechanism facilitating cross talk between the endothelium 191 and WAT. Treatment of primary human adipocytes with conditioned media from primary EC 192 of ECIGF-1R KD fed HFD for two weeks led to increased UCP-1, CIDEA, PGC1a, CYCS and 193 CD137 gene expression compared to adipocytes cultured in conditioned media from wildtype 194 littermates fed HFD for two weeks (Supplementary Figure 10B). UCP-1 and CIDEA expression 195 induced by ECIGF-1 $\mathrm{R}^{\mathrm{KD}} \mathrm{EC}$ conditioned media was preserved following protein denaturation 196 by boiling, suggesting a non-protein signal (Supplementary Figure 10C). We used a metabolomic approach to compare the small molecule secretome of EC from ECIGF-1 $\mathrm{R}^{\mathrm{KD}}$ mice and their littermate controls after two weeks of HFD and found distinct differences in the small molecule endothelial secretome (Supplementary figure $11 \mathrm{~A}$ ). To our knowledge this is the first study to demonstrate the potential importance of the endothelial small molecule secretome in the pathophysiology of obesity.

Malonic acid is a novel adipose tissue beiging metabokine

A screen of the upregulated metabolites released from ECIGF-1R ${ }^{K D} E C$ (Supplementary figure 11B) revealed that malonic acid was sufficient to upregulate Ucp-1, Cidea, Cd137, Cited1, Fgf21 and Tmem26 gene expression in 3T3-L1 adipocytes (Figure 3A). A concentration of 10mM malonic (Supplementary Figure 12) increased adiponectin secretion from 3T3-L1 adipocytes (Figure 3B). Time-course experiments demonstrated malonic acid upregulated Fgf21 gene expression first, followed by Ucp-1 and Cidea gene expression (Figure 3C).

211 It has previously been shown that malonate, the ionised form of malonic acid, can increase 212 reactive oxygen species (ROS) production ${ }^{31}$. We therefore asked if malonic acid-induced 213 beiging is mediated by ROS. To answer this, we treated adipocytes with MitoQ, a 214 mitochondrial-targeted antioxidant ${ }^{32}$, prior to malonic acid treatment. Removal of 215 mitochondrial-generated ROS by MitoQ attenuated malonic acid-induced Fgf21 gene upregulation (Figure 3D), demonstrating that malonic acid-induced upregulation of FGF21 signalling is in part dependent on ROS. 
219 FGF21 was previously reported as a paracrine/autocrine beiging mediator in WAT, enriched 220 in murine rosiglitazone-stimulated beige adipocytes and norepinephrine-stimulated brown 221 adipocytes ${ }^{33,34,3536}$. An FGF21 receptor blocker (Figure 3E) diminished malonic acid induced 222 upregulation of Ucp-1 and Cidea in 3T3-L1 adipocytes, consistent with previous studies 223 showing FGF21 regulates $U_{c p-1}{ }^{37,38}$.

224 To investigate the therapeutic potential of malonic acid as a beiging agent, we utilized various 225 malonate prodrugs $^{39}$ (Supplementary figure 13). These malonate prodrugs accelerate 226 malonate delivery in vivo ${ }^{39}$. The prodrug, di-tert-butyl malonate (DBM) induced an upregulation 227 of Cd137, Cited1, Fgf21 and Ucp-1 gene expression in 3T3-L1 adipocytes (Figure 3F) and 228 UCP-1 and CIDEA gene expression in human primary adipocytes (Figure 3G), demonstrating a novel therapeutic strategy for inducing beiging in white adipocytes.

231 Although the action of malonic acid to inhibit succinate dehydrogenase (SDH) was established 232 over 80 years $\mathrm{ago}^{40}$, our data demonstrate for the first time that, using an alternative 233 concentration and exposure time, malonic acid leads to beiging of WAT. Mills et al., ${ }^{41}$ in a

234 shivering thermogenesis model, suggested that elevated succinate led to browning of WAT in 235 a SDH and ROS dependent fashion. In contrast to our findings, Mills et al., suggested that 236 malonic acid, by inhibiting SDH, blocked the effect of succinate. However, our data raise the 237 possibility that malonic acid may act via an alternative pathway to induce adipocyte beiging in 238 WAT in a ROS/FGF21 dependent fashion (Figure 3H).

241 In conclusion, our data reveal a hitherto unrecognised non-cell autonomous paracrine 242 mechanism by which a reduction in EC IGF-1R stimulates beiging of WAT in mice challenged 243 by a high-fat diet. Moreover, we present the novel finding that malonic acid, is released by the 244 endothelium when IGF-1R levels are reduced and functions as a 'metabokine' leading to 245 beiging of white adipocytes. 
Figure 1

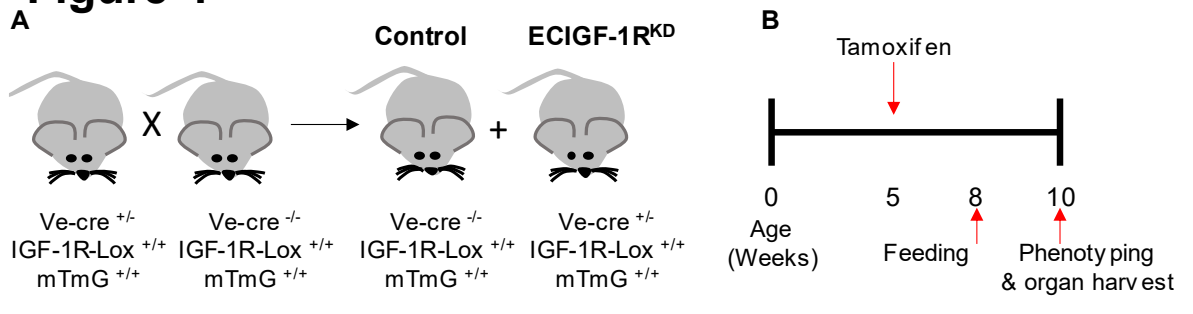

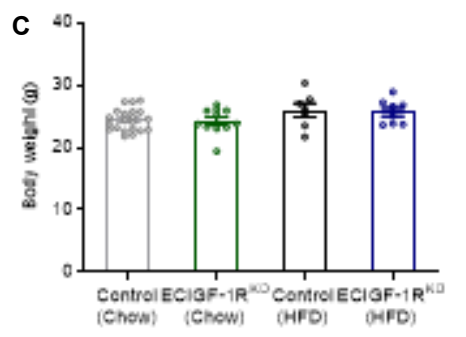

D
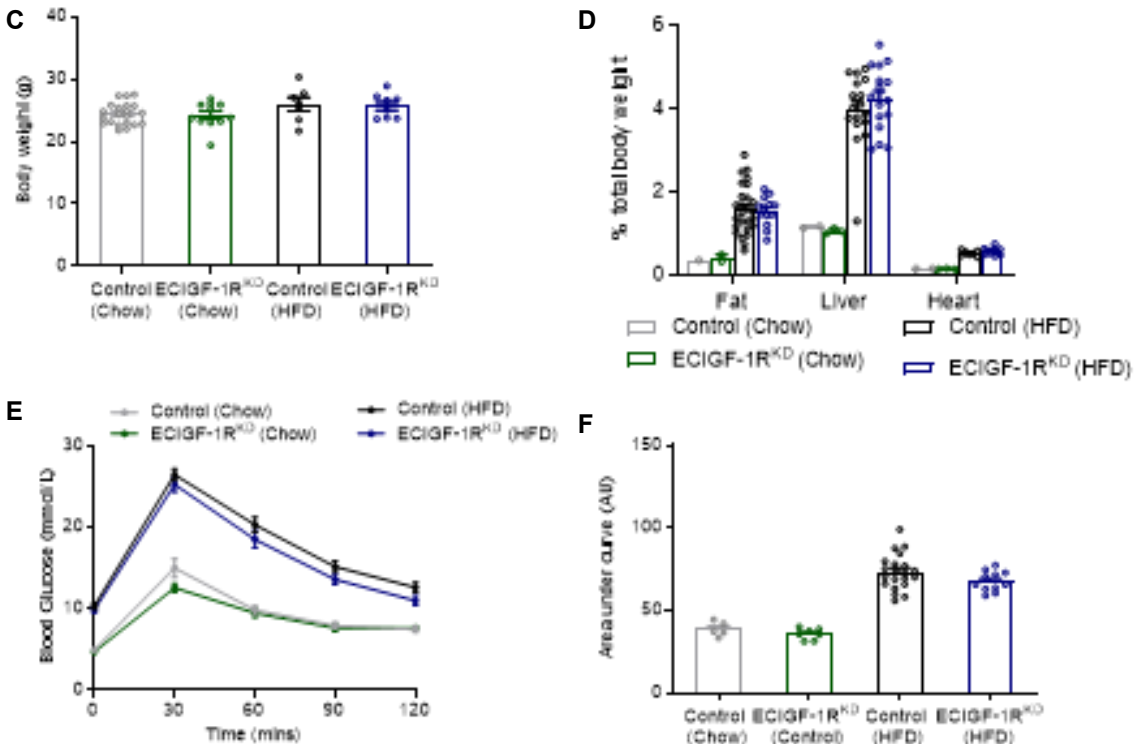

G
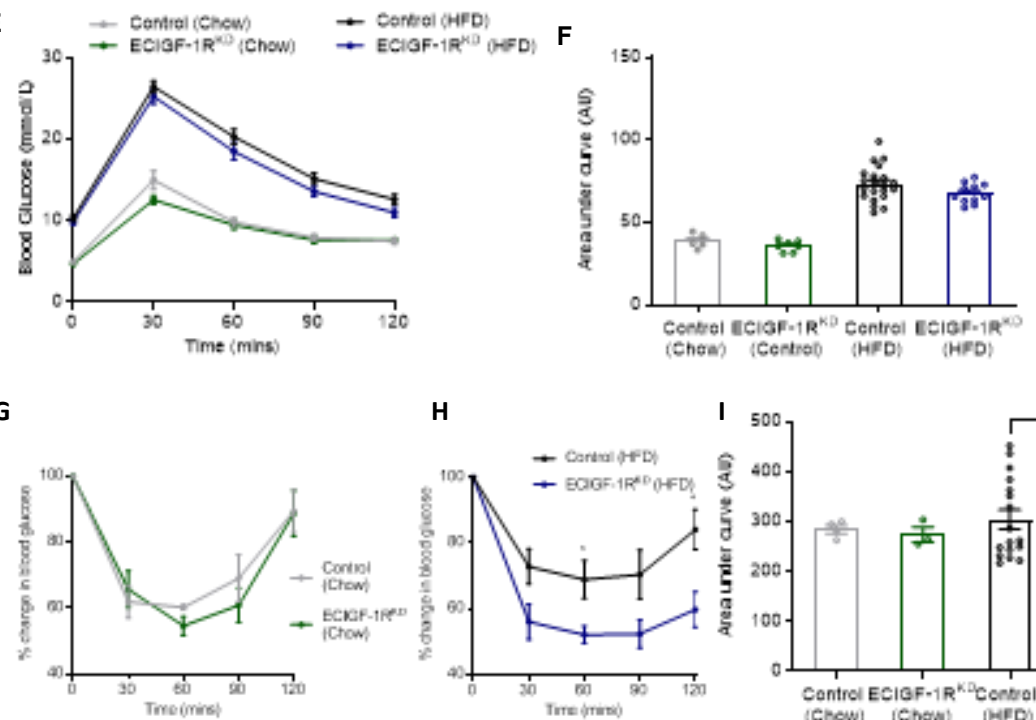

250 Figure 1 - Reduction in murine endothelial IGF-1R expression improves whole body insulin sensitivity in the setting of over nutrition

252 A. Schematic representation of the generation of tamoxifen-inducible endothelial cell specific IGF-1R knock down mice (ECIGF-1R ${ }^{\mathrm{KD}}$ ).

B. Schematic representation of experimental protocol.

C. Quantification of body weight from chow-fed control and ECIGF-1R ${ }^{\mathrm{KD}}$ mice and from 2week high fat fed (HFD) control and ECIGF-1R ${ }^{\mathrm{KD}}$ mice. ( $\mathrm{n}=$ Chow 21\&11, HFD 7 \&8).

D. Quantification of wet organ weight from chow-fed control and ECIGF-1 $R^{\mathrm{KD}}$ mice and from 2-week HFD control and ECIGF-1R ${ }^{\mathrm{KD}}$ mice ( $\mathrm{n=Chow}$ 2\&3, HFD 26 \&16).

E. Glucose tolerance over time for chow-fed control and ECIGF-1R ${ }^{\mathrm{KD}}$ mice and for 2-week HFD control and ECIGF-1 $R^{K D}$ mice ( $n=$ Chow 5\&7, HFD 21\&11). 
261 F. Area under the curve (AUC) analysis for glucose tolerance test for chow fed control and ECIGF-1 ${ }^{K D}$ mice and for 2-week HFD control and ECIGF-1 $R^{K D}$ mice ( $n=$ Chow 5\&7, HFD 21\&11).

264 G. Insulin tolerance test for chow-fed control and ECIGF-1 $R^{K D}$ mice $(n=4 \& 3)$.

265 H. Insulin tolerance test for 2-week HFD control and ECIGF-1R ${ }^{K D}$ mice $(n=17 \& 10)$.

266 I. The area under the curve analysis for insulin tolerance tests for chow-fed control and ECIGF-1R ${ }^{K D}$ mice and from 2-week HFD control and ECIGF-1 $R^{K D}$ mice ( $\left.n=4,3,17 \& 10\right)$.

Data shown as mean \pm SEM, data points are individual mice. $p<0.05$ taken as statistically significant using student unpaired two tailed t-test and denoted as *. 


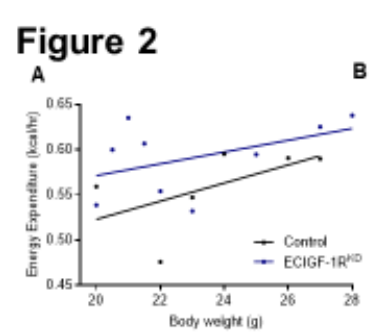

D
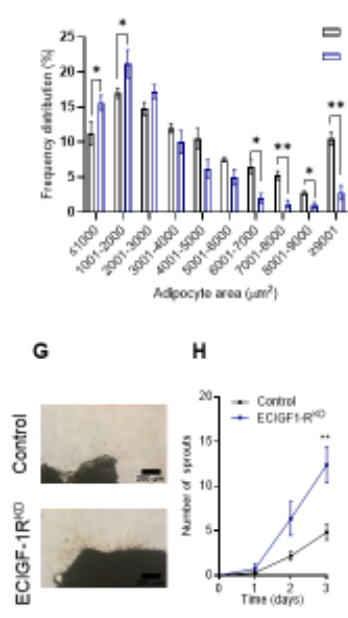

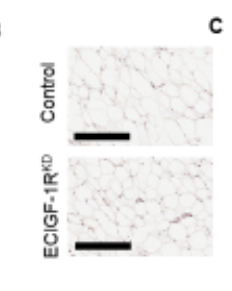

E

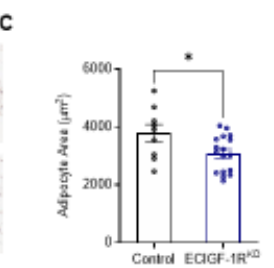

F
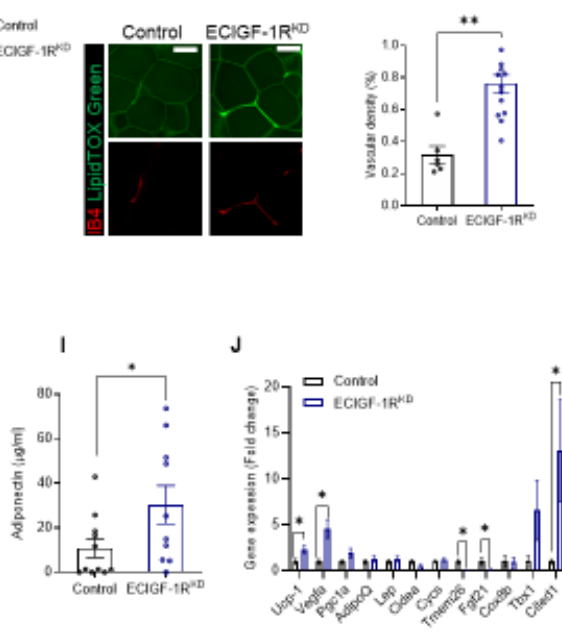

Figure 2 - Reduction in murine endothelial IGF-1R expression prevents deleterious remodelling of adipose tissue in the setting of over nutrition

A. Energy expenditure in 2-week HFD fed control and ECIGF-1 $R^{K D}$ mice ( $\left.n=6 \& 9\right)$.

B. Representative images of hematoxylin and eosin ( $\mathrm{H} \& \mathrm{E})$ stained white epididymal adipose tissue from 2-week HFD control and ECIGF-1R ${ }^{K D}$ mice (Scale bar $=200 \mu \mathrm{m}$ ).

C. Quantification of adipocyte size from 2-week HFD control and ECIGF-1R ${ }^{K D}$ mice ( $n$ $=9 \& 17)$.

D. Quantification of white epididymal adipocyte size distribution from 2-week HFD control and ECIGF-1R ${ }^{K D}$ mice ( $\left.n=9 \& 17\right)$.

E. Representative images of isolectin B4 (Red) and LipidTox (Green) stained white epididymal adipose tissue from 2-week HFD control and ECIGF-1R ${ }^{K D}$ mice (Scale bar $=100 \mu \mathrm{m})$.

F. Quantification of white epididymal adipose tissue vascularisation from 2-week HFD control and ECIGF-1R ${ }^{\mathrm{KD}}$ mice $(\mathrm{n}=6 \& 14)$.

G. Representative images of 2-week HFD control and ECIGF-1 $\mathrm{R}^{\mathrm{KD}}$ white epididymal adipose tissue explants (Scale bar $=200 \mu \mathrm{m})$. 
290 H. Quantification of white epididymal adipose tissue neovascularisation from 2-week HFD 291 control and ECIGF-1R ${ }^{\mathrm{KD}}$ mice $(\mathrm{n}=5 \& 5)$.

292 I. Quantification of plasma adiponectin levels from 2-week HFD control and ECIGF-1R $\mathrm{R}^{\mathrm{KD}}$ 293 mice $(n=10 \& 11)$.

294 J. Quantitation of white epididymal adipose gene expression from 2-week HFD control and ECIGF-1 ${ }^{K D}$ mice ( $\left.n=9-17\right)$.

296

297 Data shown as mean \pm SEM, data points are individual mice. $p<0.05$ taken as statistically significant using student unpaired two tailed t-test and denoted as ${ }^{*}\left({ }^{* *} p \leq 0.01\right)$. 


\section{Figure 3}
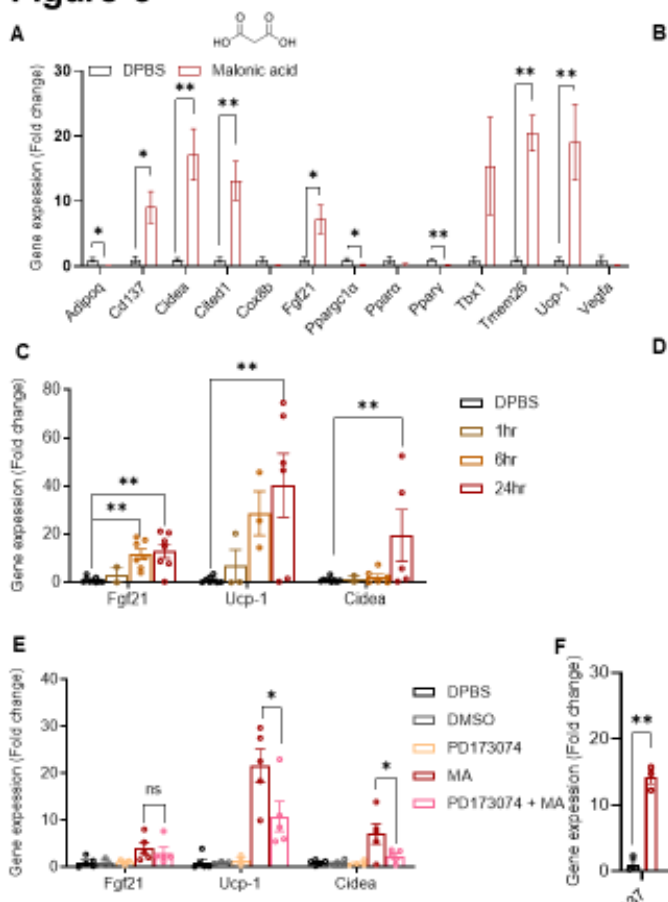

G

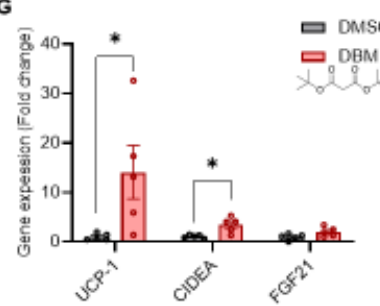

口 DMSO

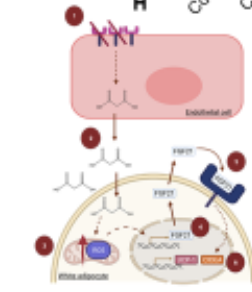

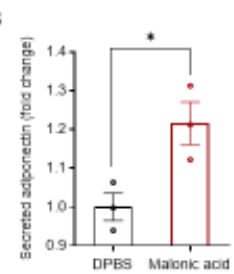
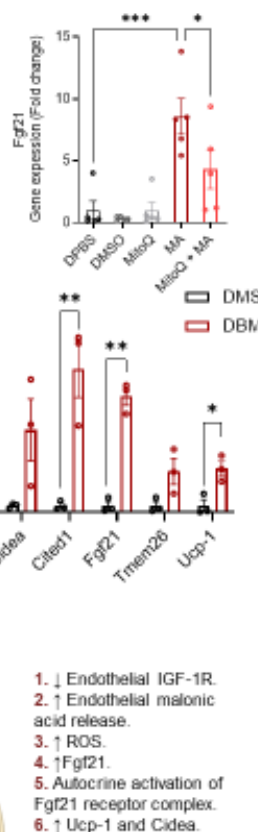

301

Figure 3 - Reduction in murine endothelial IGF-1R expression alters the endothelial secretome and reveals a role for malonic acid in modulating white adipose function

A. Quantification of gene expression in 3T3-L1 adipocytes after $24 \mathrm{hr} 10 \mathrm{mM}$ malonic acid stimulation ( $n=4-7$ per treatment).

B. Quantification of adiponectin secretion in 3T3-L1 adipocytes after $24 \mathrm{hr} 10 \mathrm{mM}$ malonic acid stimulation ( $n=3$ per treatment group).

C. Quantification of gene expression in 3T3-L1 adipocytes after varying exposure times to $10 \mathrm{mM}$ malonic acid stimulation ( $\mathrm{n}=4-7$ per treatment group).

D. Quantification of Fgf21 gene expression in 3T3 -L1 adipocytes, after treatment with mitoQ and malonic acid $10 \mathrm{mM}$ for $24 \mathrm{hrs}$ ( $\mathrm{n}=5$ per treatment group).

E. Quantification of Ffg21, Ucp-1 and Cidea gene expression in 3T3-L1 adipocytes after treatment with FGF1R blocker (PD17304) and malonic acid 10mM for 24hrs ( $n=3-5$ per treatment group).

F. Quantification of gene expression in 3T3-L1 adipocytes after $24 \mathrm{hr} 10 \mathrm{mM}$ di-tert-butyl malonate (DBM) stimulation ( $\mathrm{n}=3$ per treatment group).

G. Quantification of gene expression in human primary adipocytes after $24 \mathrm{hr} 10 \mathrm{mM}$ di-tertbutyl malonate (DBM) stimulation ( $n=5$ per treatment group). 
319 H. Schematic diagram of the proposed mechanism of EC IGF-1R mediated white adipocyte beiging.

Data shown as mean \pm SEM, $n$ is an individual experiment. $p<0.05$ taken statistically significant using student unpaired two tailed t-test or ANOVA and denoted as * $(p \leq 0.01$ and is denoted as ${ }^{* *}$ ).

325

326 


\section{S Fig 1}

A

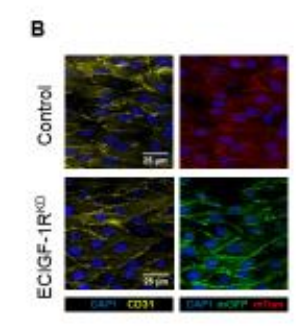

c
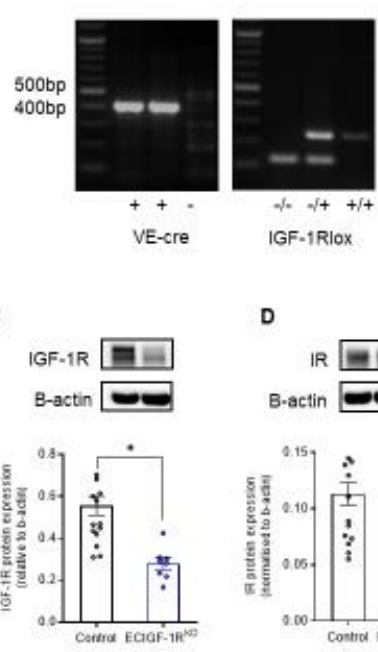

D
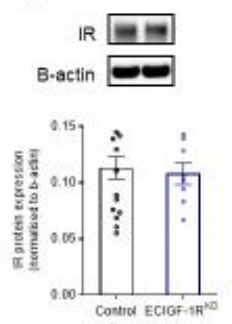

Supplementary figure 1 - Confirmation of IGF-1R reduction in murine model of endothelial specific IGF-1R knockdown

A. Representative images of genotyping during the generation of ECIGF-1R ${ }^{\mathrm{KD}}$.

B. Representative images of en face stained femoral arteries from tamoxifen-induced ECIGF$1 \mathrm{R}^{\mathrm{KD}}$ mice and control littermates confirming a switch from $\mathrm{mT}$ to $\mathrm{mG}$ with tamoxifen (Scale bar $=25 \mu \mathrm{m})$.

C. Quantitation of primary murine endothelial cell expression of IGF-1R from 2-week HFD control and ECIGF-1 $R^{\mathrm{KD}}$ mice $(\mathrm{n}=16 \& 8)$.

D. Quantitation of primary murine endothelial cell expression of insulin receptor (IR) from 2week HFD control and ECIGF-1R ${ }^{\mathrm{KD}}$ mice $(\mathrm{n}=16 \& 8)$. significant using student unpaired two tailed t-test and denoted as *. 


\section{S Fig 2}
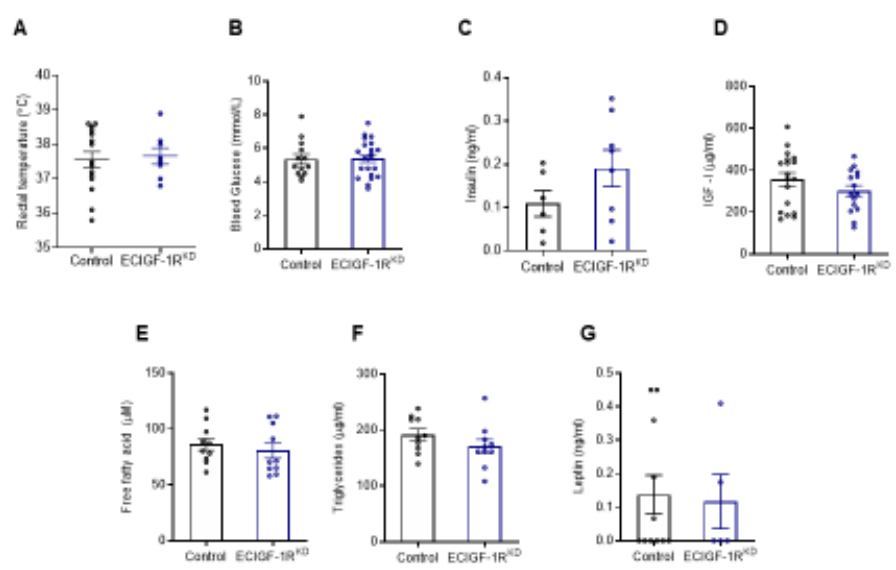

Supplementary figure 2 - No difference in metabolic plasma markers from mice with endothelial specific IGF-1R reduction in the setting of over nutrition

347 A. Core body temperature of 2-week HFD control and ECIGF-1R ${ }^{\mathrm{KD}}$ mice $(n=15 \& 8)$.

B. Fasting blood glucose levels of 2-week HFD control and ECIGF-1R ${ }^{K D}$ mice $(n=13 \& 21)$.

C. Fasting plasma insulin levels from 2-week HFD control and ECIGF-1 $R^{\mathrm{KD}}$ mice $(n=6 \& 8)$.

D. Fasting circulating plasma IGF-1 levels from 2-week HFD control and ECIGF-1R ${ }^{\mathrm{KD}}$ mice $(n=18 \& 15)$.

E. Fasting plasma free fatty acids levels from 2-week HFD control and ECIGF-1R ${ }^{K D}$ mice ( $n$ $=10 \& 10$ ).

F. Fasting plasma triglyceride levels from 2-week HFD control and ECIGF-1R ${ }^{\mathrm{KD}}$ mice ( $\mathrm{n}$ $=10 \& 10$ ).

G. Fasting plasma leptin levels of 2-week HFD control and ECIGF-1R ${ }^{\mathrm{KD}}$ mice $(n=11 \& 5)$. statistically significant using student unpaired two tailed t-test and denoted as *. 
S fig 3
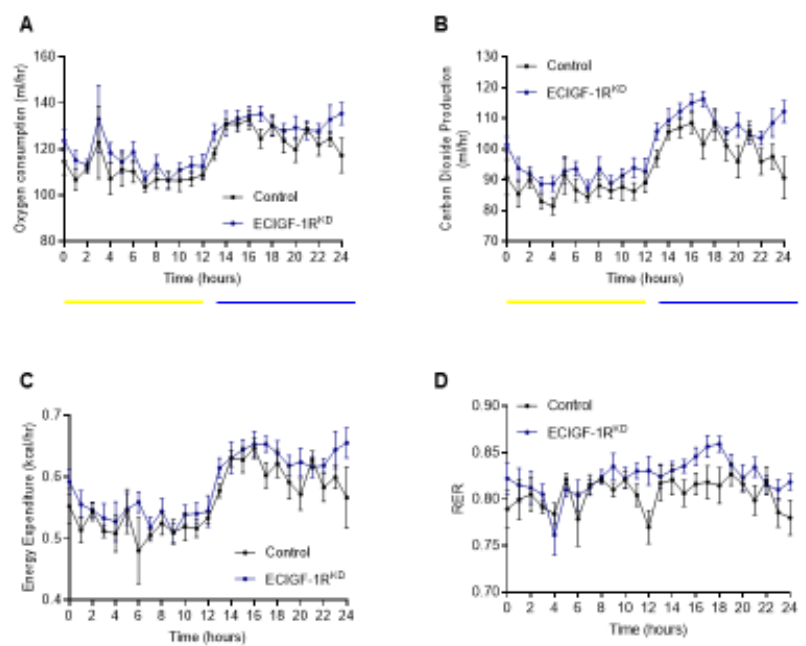

D
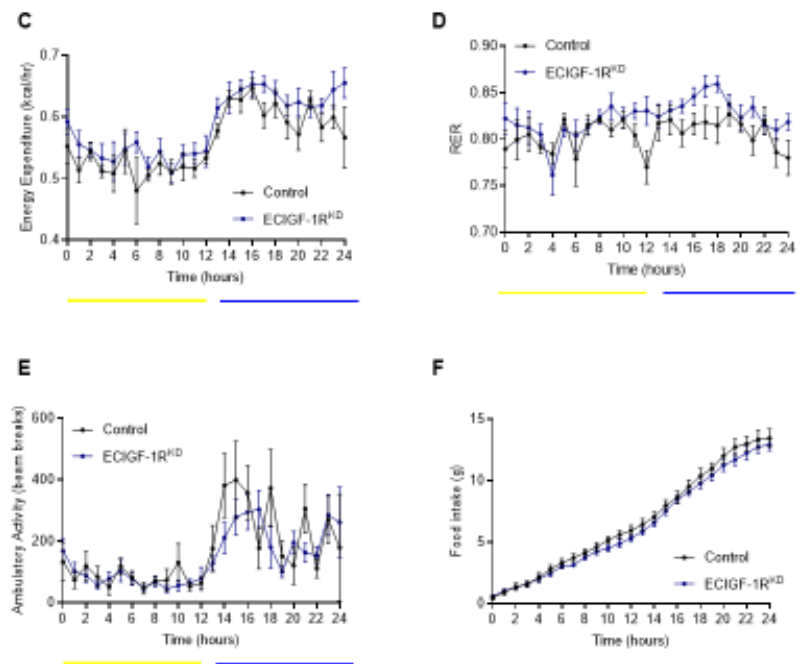

$\mathbf{F}$

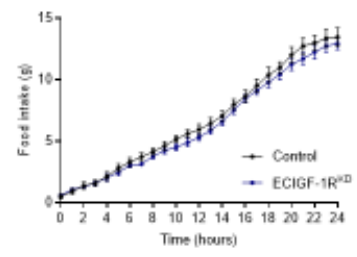

Supplementary figure 3 - Characterisation of energy expenditure in mice with reduced endothelial IGF-1R expression after 2 weeks of high fat diet

364 A. Oxygen consumption for 2-week HFD control and ECIGF-1R ${ }^{K D}$ mice $(n=6 \& 9)$.

B. Carbon dioxide production for 2-week HFD control and ECIGF-1R $R^{K D}$ mice $(n=6 \& 9)$.

C. Energy expenditure for 2-week HFD control and ECIGF-1R ${ }^{K D}$ mice $(n=6 \& 9)$.

D. Respiratory exchange ratio for 2-week HFD control and ECIGF-1R ${ }^{K D}$ mice $(n=6 \& 9)$.

E. Activity levels for 2-week HFD control and ECIGF-1R ${ }^{K D}$ mice $(n=6 \& 9)$.

F. Cumulative food consumption for 2-week HFD control and ECIGF-1 $R^{K D}$ mice $(n=6 \& 9)$.

The light/dark cycle for graphs A-E are shown as follows; Light in yellow and dark in blue. Data shown as mean \pm SEM, $p<0.05$ taken as being statistically significant using student t-test and denoted as *. Metabolic parameters were measured by indirect calorimetry, ANOVA testing was performed using mass as a co-variant (ANCOVA testing) using calrapp.org. 


\section{S Fig 4}
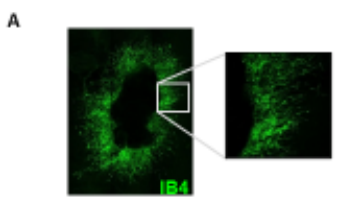

379

380 Supplementary figure 4 - Confirming endothelial cells in neovascularisation

381 A. Staining of adipose tissue explants confirms sprouts are endothelial with positive isolectin $382 \quad$ B4 staining (green).

383 

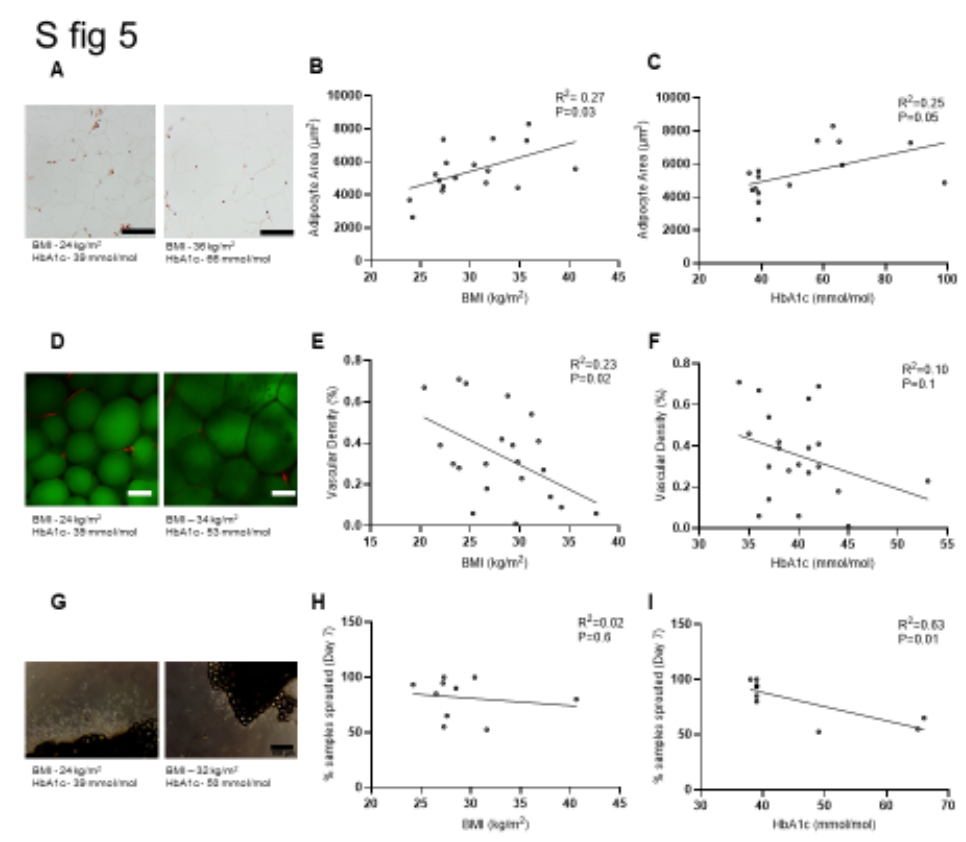
and HbA1c.

388 A. Representative images of hematoxylin and eosin (H \& E)-stained white subcutaneous adipose tissue from patients with lower $\mathrm{BMI}$ and $\mathrm{HbA1C}$ and with higher $\mathrm{BMI}$ and $\mathrm{HbA} 1 \mathrm{C}$ (Scale bar $=200 \mu \mathrm{m})$.

B. Correlation between BMI and adipocyte area ( $\mathrm{n}=17$ patients).

C. Correlation between $\mathrm{HbA} 1 \mathrm{C}$ and adipocyte area ( $\mathrm{n}=17$ patients).

D. Representative images of Ulex Europaeus (Red) and LipidTox (Green) stained white subcutaneous adipose tissue from patients with lower BMI and $\mathrm{HbA1C}$ and with higher $\mathrm{BMI}$ and $\mathrm{HbA1c}$ (Scale bar $=100 \mu \mathrm{m}$ ).

396 E. Correlation between BMI and adipose vascularity ( $n=21$ patients).

397 F. Correlation between HbA1C and adipose vascularity ( $n=21$ patients).

398 G. Representative images of human white subcutaneous adipose tissue from patients with lower BMI and $\mathrm{HbA1C}$ and with higher BMl and HbA1c. (Scale bar $=100 \mu \mathrm{m}$ ).

400

H. Correlation between BMI and adipose neovascularisation ( $n=10$ patients).

401

I. Correlation between $\mathrm{HbA1C}$ and adipose neovascularisation ( $\mathrm{n}=10$ patients). 
bioRxiv preprint doi: https://doi.org/10.1101/2021.12.01.470734; this version posted December 1, 2021. The copyright holder for this preprint (which was not certified by peer review) is the author/funder. All rights reserved. No reuse allowed without permission. to assess the degree of relation between $\mathrm{BMI}$ and $\mathrm{Hba1C}$ and various fat markers. $\mathrm{p}<0.05$

405 taken as statistically significant.

406 


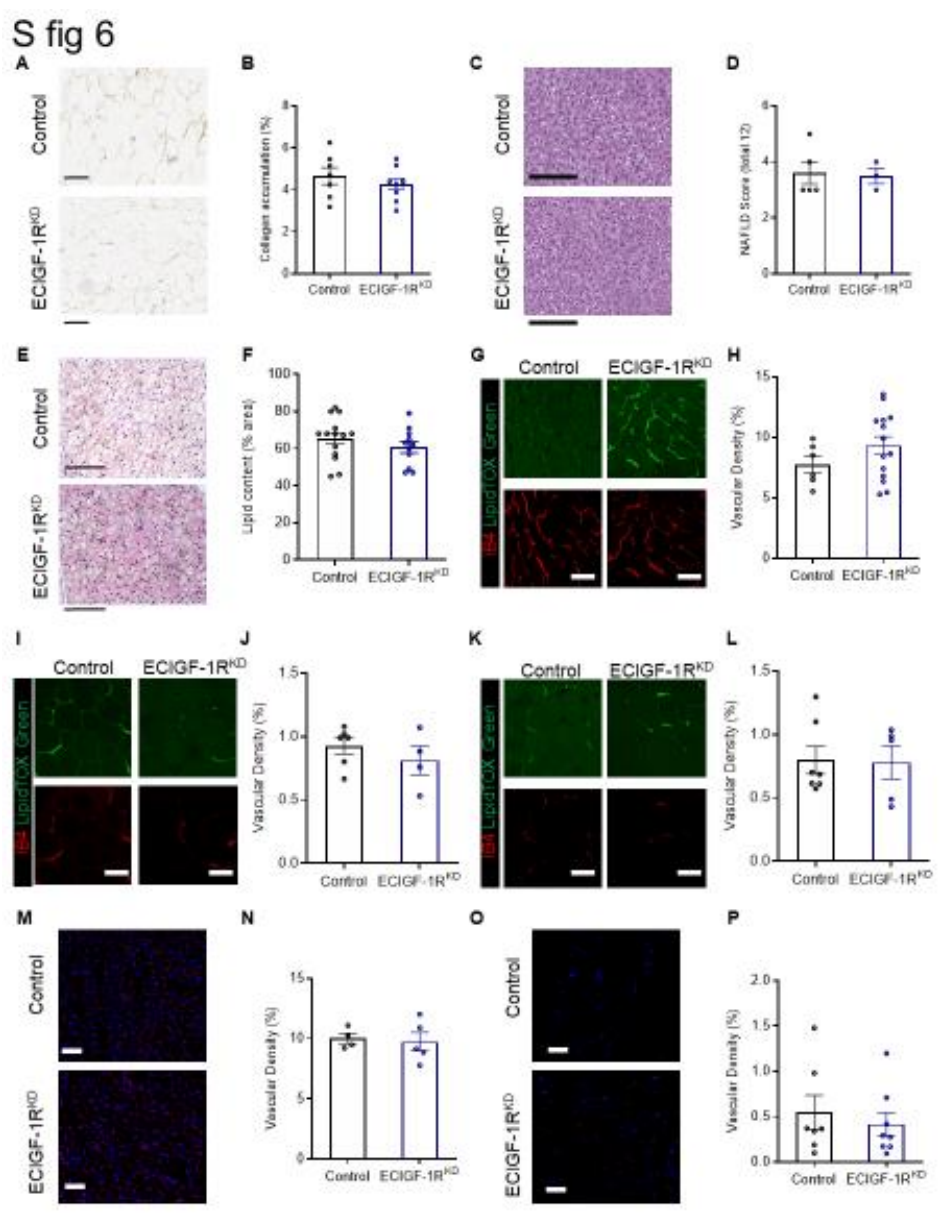
IGF-1R expression after 2 weeks of high fat diet

411 A. Representative images of picro sirius red stained white epididymal adipose tissue from 2week HFD control and ECIGF-1 $\mathrm{R}^{\mathrm{KD}}$ mice (Scale bar $\left.=200 \mu \mathrm{m}\right)$.

413 B. Quantification of white epididymal adipose collagen deposition from 2-week HFD control and ECIGF-1R $\mathrm{R}^{\mathrm{KD}}$ mice $(\mathrm{n}=7 \& 9)$.

C. Representative images of Hematoxylin and eosin ( $\mathrm{H}$ and $\mathrm{E}$ )-stained liver from 2-week HFD control and ECIGF-1 $R^{K D}$ mice (Scale bar $\left.=200 \mu \mathrm{m}\right)$.

D. Quantification of non-alcoholic fatty liver disease (NAFLD) from 2-week HFD control and ECIGF-1R ${ }^{\mathrm{KD}}$ mice $(\mathrm{n}=5 \& 3)$.

419 E. Representative images of $\mathrm{H}$ and E-stained brown interscapular adipose tissue from 2week HFD control and ECIGF-1R ${ }^{\mathrm{KD}}$ mice (Scale bar $\left.=100 \mu \mathrm{m}\right)$.

F. Quantification of lipid content of interscapular brown adipose tissue from 2-week HFD control and ECIGF-1R $\mathrm{R}^{\mathrm{KD}}$ mice $(\mathrm{n}=14 \& 12)$.

G. Representative images of isolectin B4 (Red) and LipidTox (Green) stained brown interscapular adipose tissue from 2-week HFD control and ECIGF-1 $\mathrm{R}^{\mathrm{KD}}$ mice (Scale bar = $100 \mu \mathrm{m})$. 
H. Quantification of vascularity in interscapular brown adipose tissue from 2-week HFD control and ECIGF-1 $\mathrm{R}^{\mathrm{KD}}$ mice ( $\left.\mathrm{n}=6 \& 14\right)$.

I. Representative images of isolectin B4 (Red) and LipidTox (Green) stained white subcutaneous adipose tissue from 2-week HFD control and ECIGF-1R ${ }^{\mathrm{KD}}$ mice (Scale bar $=100 \mu \mathrm{m})$.

J. Quantification of vascularity in white subcutaneous adipose tissue from 2-week HFD control and ECIGF-1R ${ }^{\mathrm{KD}}$ mice $(\mathrm{n}=6 \& 4)$.

K. Representative images of isolectin B4 (Red) and LipidTox (Green) stained white perinephric adipose tissue from 2-week HFD control and ECIGF-1 $\mathrm{R}^{\mathrm{KD}}$ mice (Scale bar = $100 \mu \mathrm{m})$.

L. Quantification of vascularity in white perinephric adipose tissue from 2-week HFD control and ECIGF-1R $\mathrm{R}^{\mathrm{KD}}$ mice ( $\left.\mathrm{n}=7 \& 5\right)$.

M. Representative images of isolectin B4 (Red) and DAPI (Blue) stained liver from 2-week HFD control and ECIGF-1R $\mathrm{R}^{\mathrm{KD}}$ mice (Scale bar $\left.=50 \mu \mathrm{m}\right)$.

440 N. Quantification of liver vascularisation from 2-week HFD control and ECIGF-1R ${ }^{K D}$ mice (n $=4 \& 5$ ).

O. Representative images of isolectin B4 (Red) and DAPI (Blue) stained muscle from 2-week HFD control and ECIGF-1R $\mathrm{RD}^{\mathrm{KD}}$ mice (Scale bar $\left.=50 \mu \mathrm{m}\right)$.

P. Quantification of muscle vascularisation from 2-week HFD control and ECIGF-1R ${ }^{\mathrm{KD}}$ mice $(\mathrm{n}=7 \& 8)$. 
S fig 7

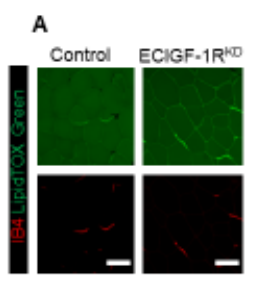

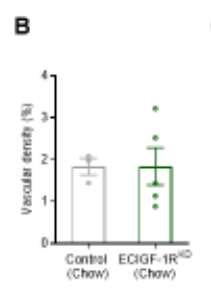

C

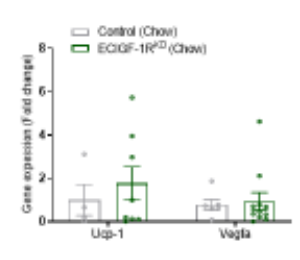

452

453 Supplementary figure 7 - Characterisation of adipose tissue from chow fed mice with 454 reduced endothelial IGF-1R expression

455 A. Representative images of isolectin B4 (Red) and LipidTox (Green) stained white 456 epididymal adipose tissue from chow fed control and ECIGF-1R $\mathrm{R}^{\mathrm{KD}}$ mice (Scale bar = $457 \quad 100 \mu \mathrm{m})$.

458 B. Quantification of vascularity in white epididymal adipose tissue from chow fed control and ECIGF-1R ${ }^{\mathrm{KD}}$ mice ( $\left.\mathrm{n}=3 \& 5\right)$.

460 C. Quantification of Ucp-1 and Vegfa gene expression in white epididymal adipose tissue from chow fed control and ECIGF-1 $R^{\mathrm{KD}}$ mice $(\mathrm{n}=4-11)$.

462

Data shown as mean \pm SEM, Individual mice are shown as separate datapoints $p<0.05$ 
S fig 8
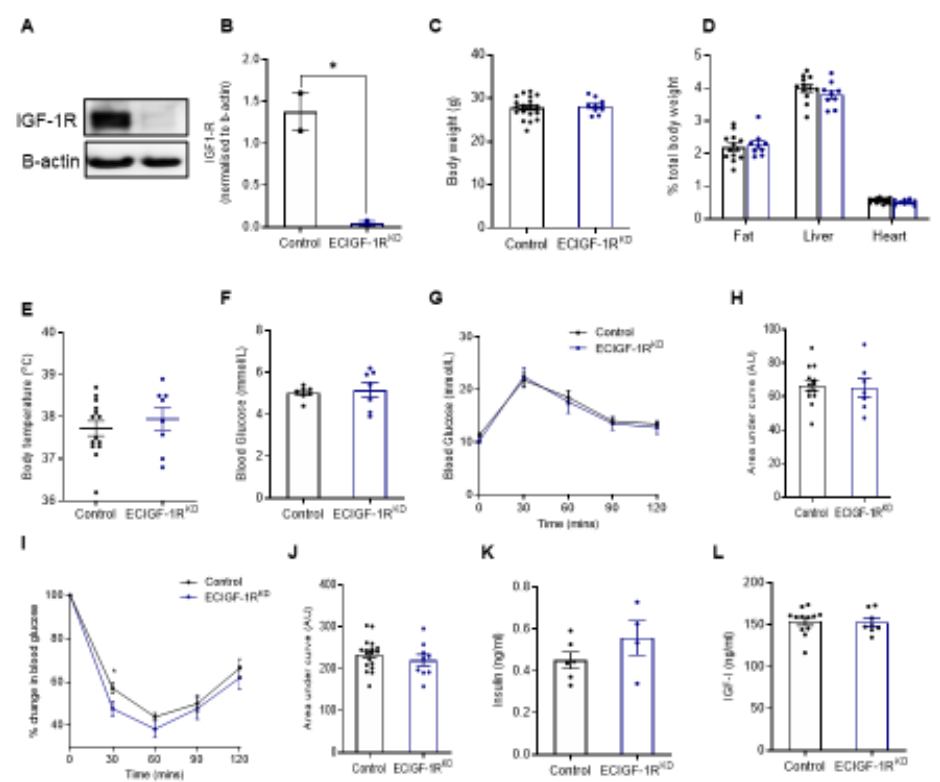

468

Supplementary figure 8 - Metabolic characterisation of mice with reduced endothelial IGF-1R expression after 8-weeks high fat diet.

471 A. Representative western blot of primary murine endothelial cell expression of IGF-1R from 8-week HFD fed control and ECIGF-1 $\mathrm{R}^{\mathrm{KD}}$ mice.

B. Quantitation of primary murine endothelial cell expression of IGF-1R from 8-week HFD control and ECIGF-1 $R^{\mathrm{KD}}$ mice ( $\left.\mathrm{n}=2 \& 2\right)$.

C. Body mass of 8-week HFD fed control and ECIGF-1R ${ }^{K D}$ mice ( $\left.n=20 \& 10\right)$.

D. Wet organ weight of 8-week HFD control and ECIGF-1R ${ }^{\mathrm{KD}}$ mice $(n=13 \& 9)$.

E. Core body temperature of 8-week HFD fed control and ECIGF-1 $R^{K D}$ mice $(n=14 \& 8)$.

F. Fasting blood glucose levels from 8-week HFD fed control and ECIGF-1 $R^{\mathrm{KD}}$ mice ( $\left.n=7 \& 7\right)$

G. Glucose tolerance over time of 8-week HFD fed control and ECIGF-1R ${ }^{\mathrm{KD}}$ mice $(n=13 \& 7)$.

480 H. Area under the curve (AUC) analysis of glucose tolerance of 8-week HFD fed control and ECIGF-1 $R^{\mathrm{KD}}$ mice $(\mathrm{n}=13 \& 7)$.

I. Insulin tolerance over time of 8-week HFD fed control and ECIGF-1R ${ }^{\mathrm{KD}}$ mice ( $\mathrm{N}=18$ \&11).

J. Area under the curve (AUC) analysis of insulin tolerance test of 8-week HFD fed control and ECIGF-1 $\mathrm{R}^{\mathrm{KD}}$ mice $(\mathrm{n}=18 \& 11)$.

K. Fasting plasma insulin levels from 8-week HFD fed control and ECIGF-1R ${ }^{K D}$ mice $(n=6 \& 4)$. 
L. Fasting plasma IGF-1 levels from 8-week HFD fed control and ECIGF-1R ${ }^{\mathrm{KD}}$ mice 487 $(n=12 \& 8)$.

488

489

Data shown as mean \pm SEM, individual mice are shown as separate datapoints $p<0.05$

490 taken as being statistically significant using a student unpaired two tailed t-test and

491 denoted as *.

492

493 


\section{S fig 9}
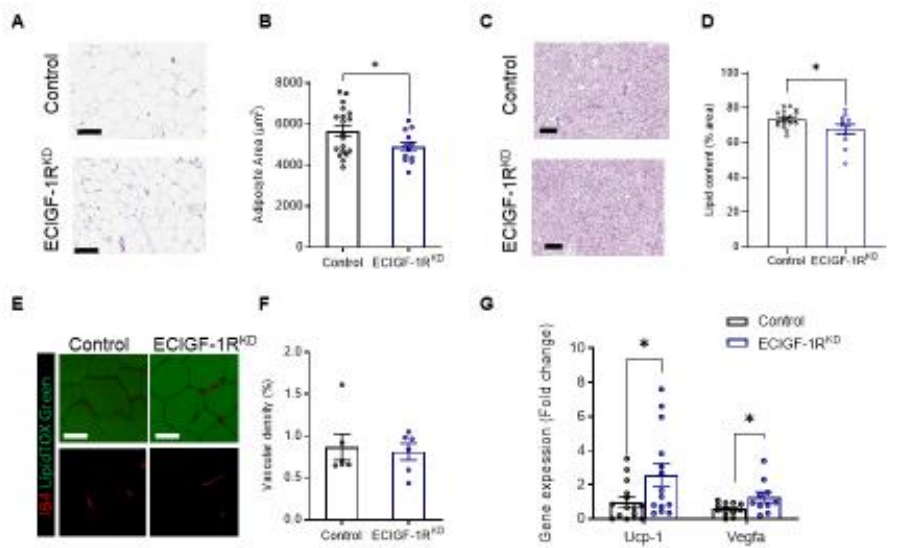

c
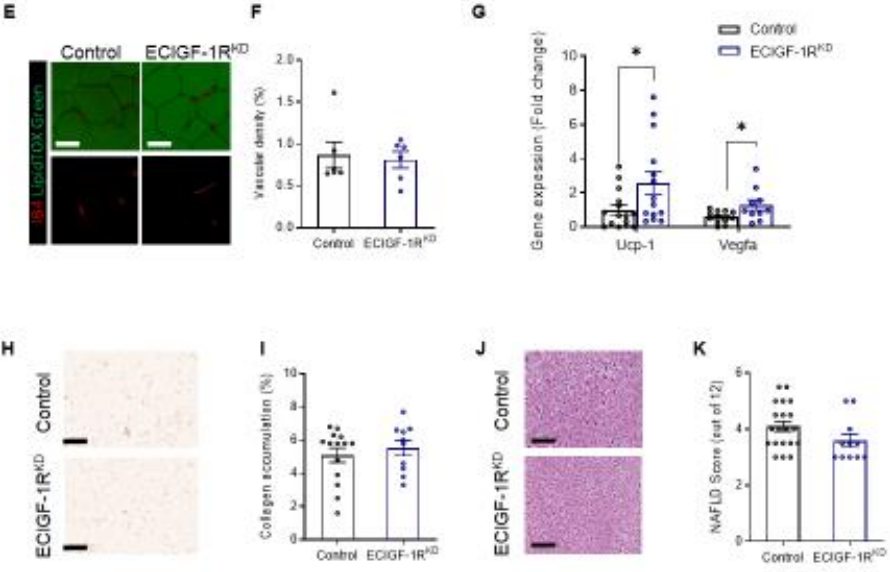

494

Supplementary figure 9 - Histological characterisation of mice with reduced endothelial IGF-1R expression after 8-weeks of high fat diet.

A. Representative images of Hematoxylin and eosin (H \& E) stained white epididymal adipose tissue from 8-week HFD fed control and ECIGF-1R ${ }^{\mathrm{KD}}$ mice (Scale bar $=200 \mu \mathrm{m}$ ).

B. Quantification of adipocyte size from 8-week HFD fed control and ECIGF-1R ${ }^{K D}$ mice ( $n=$ 12-18).

C. Representative images of $\mathrm{H} \& \mathrm{E}$ stained brown interscapular adipose tissue from 8-week HFD fed control and ECIGF-1 $R^{\mathrm{KD}}$ mice (Scale bar $=200 \mu \mathrm{m}$ ).

D. Quantification of lipid content of brown interscapular adipose tissue from 8-week HFD fed control and ECIGF-1 $R^{\mathrm{KD}}$ mice $(\mathrm{n}=18 \& 13)$.

E. Representative images of isolectin B4 (Red) and LipidTox (Green) stained white epididymal adipose tissue from 8-week HFD control and ECIGF-1R ${ }^{\mathrm{KD}}$ mice $($ Scale bar $=$ $100 \mu \mathrm{m})$.

508 F. Quantification of white epididymal adipose tissue vascularisation from 8-week HFD fed control and ECIGF-1 $\mathrm{R}^{\mathrm{KD}}$ mice ( $\left.\mathrm{n}=6 \& 6\right)$.

G. Quantitation of white epididymal adipose gene expression of Ucp-1 and Vegfa from 8week HFD fed control and ECIGF-1 $R^{\mathrm{KD}}$ mice. $(n=12-15)$. 
512 H. Representative images of picro sirius red stained white adipose tissue from 8-week HFD 513 fed control and ECIGF-1R ${ }^{\mathrm{KD}}$ mice (Scale bar $\left.=200 \mu \mathrm{m}\right)$.

514 I. Quantification of white epididymal adipose collagen deposition from 8-week HFD fed 515 control and ECIGF-1R $\mathrm{K}^{\mathrm{KD}}$ mice $(\mathrm{n}=14 \& 10)$.

516 J. Representative images of $\mathrm{H}$ and E-stained liver from 8-week HFD fed control and ECIGF$517 \quad 1 \mathrm{R}^{\mathrm{KD}}$ mice (Scale bar $\left.=200 \mu \mathrm{m}\right)$.

518 K. Quantification of non-alcoholic fatty liver disease (NAFLD) from 8-week HFD fed control and ECIGF-1R ${ }^{K D}$ mice $(n=20 \& 11)$. taken as being statistically significant using a student unpaired two tailed t-test and denoted as *. 
S fig 10
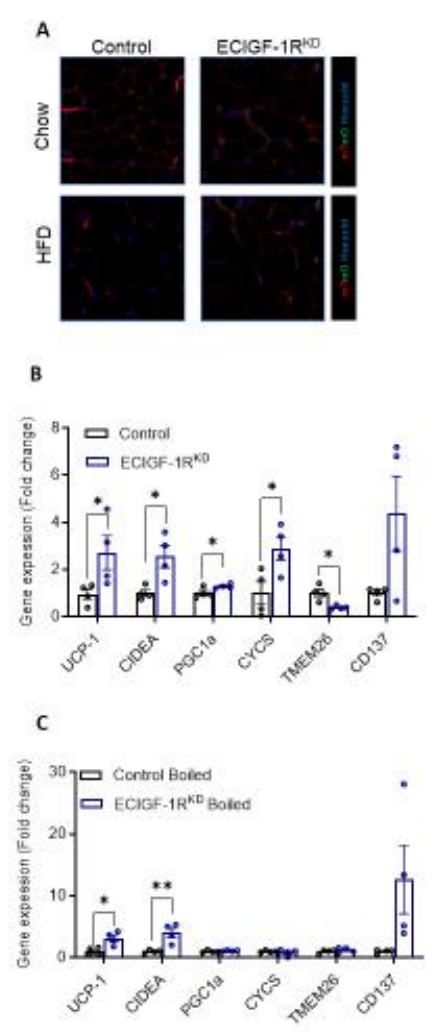

Supplementary figure 10 - Reduction in murine endothelial IGF-1R expression alters the endothelial secretome and reveals a role for a small molecule in modulating adipocyte function

A. Following induction with tamoxifen, cells of endothelial cell lineage in the ECIGF-1RKD fluoresce green using the mTmG system, with all other cells fluorescing red. All adipocytes from both genotypes appear red, confirming adipocytes are not from endothelial lineage. Quantitation of human primary adipocyte gene expression after $24 \mathrm{hr}$ treatment with conditioned media from primary murine endothelial cell isolated from 2-week HFD control and ECIGF-1R $\mathrm{R}^{\mathrm{KD}}$ mice.

B. Quantitation of human primary adipocyte gene expression after treatment with boiled conditioned media from primary murine endothelial cells isolated from 2-week HFD fed control and ECIGF-1 $R^{K D}$ mice ( $\left.n=4 \& 4\right)$.

Data shown as mean \pm SEM, individual mice are shown as separate datapoints $p<0.05$ taken as being statistically significant using a student unpaired two tailed t-test and denoted as *. 


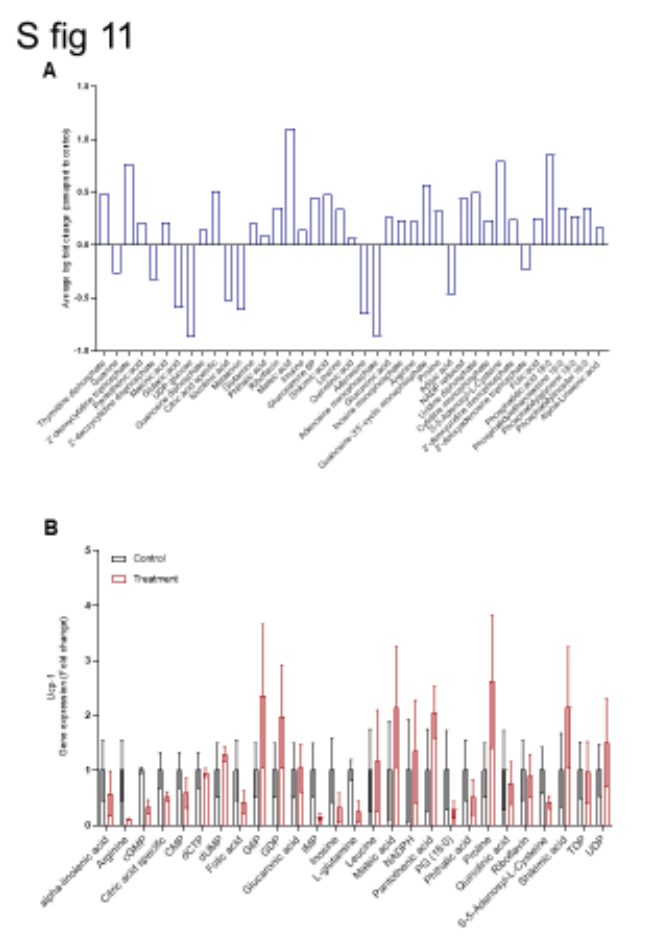

545 Supplementary figure 11 - Mice with reduced endothelial IGF-1R expression after 2546 weeks high fat diet have an altered endothelial small molecule secretome.

547 A. Small molecule analysis of the aqueous and lipid fractions of conditioned media from primary murine endothelial cell from 2-week HFD fed control and ECIGF-1 $R^{\mathrm{KD}}$ mice ( $n=4 \& 4$ per genotype).

550

B. Quantitation of 3T3-L1 adipocyte gene expression of Ucp-1 after upregulated metabolite stimulation ( $n=3-5$ per treatment group). significant using student t-test and denoted as * 
S figure 12

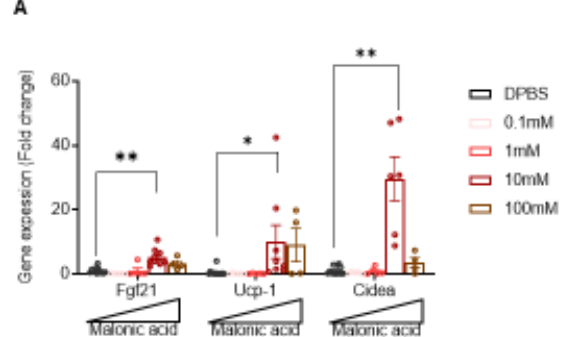

558 Supplementary figure 12 - Malonic acid dose response

559 A. Quantification of gene expression of 3T3-L1 adipocytes treated with varying doses of malonic acid for $24 \mathrm{hrs}$ ( $\mathrm{n}=5-14$ per treatment group).

Data shown as mean \pm SEM, $\mathrm{n}$ is experimental replicates $\mathrm{p}<0.05$ taken as being as $\left.{ }^{* *}\right)$.

565 


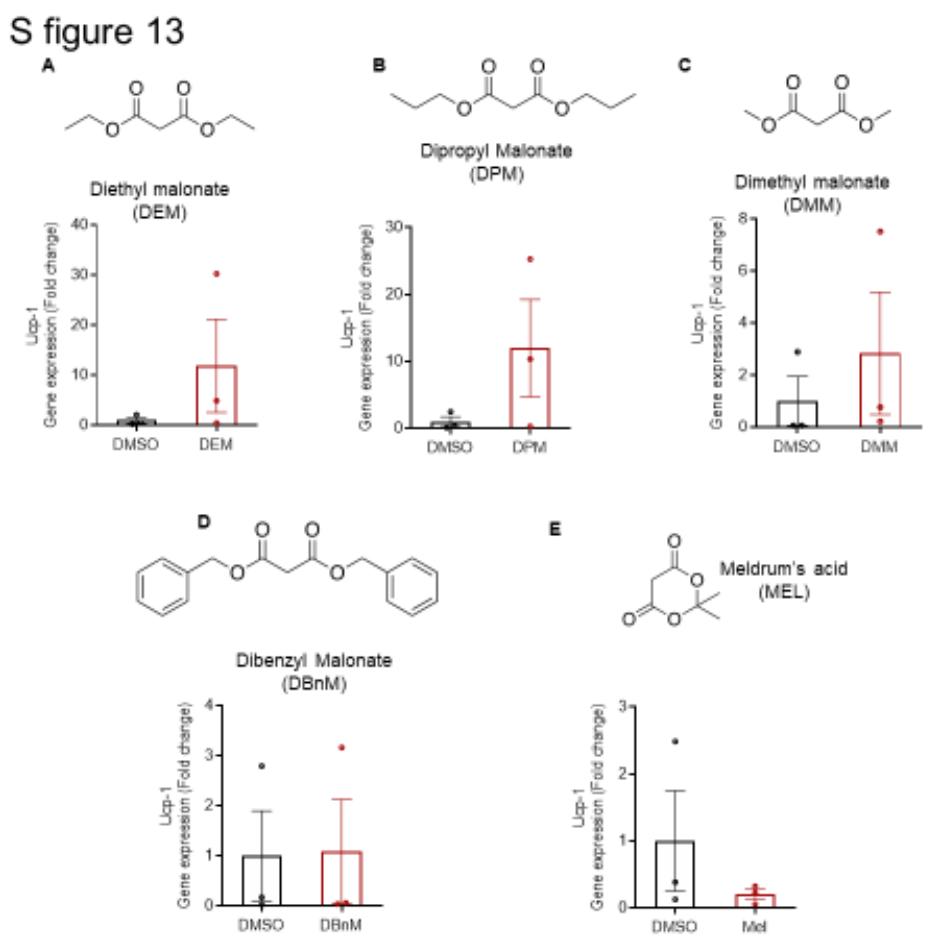

568 Supplementary figure 13 - Screening of malonic acid pro-drugs in 3T3-L1 adipocytes.

569 A. Quantification of $U c p-1$ gene expression for $24 \mathrm{hrs}$ treatment with $10 \mathrm{mM}$ Diethyl malonate (DEM) (Chemical structure shown above). ( $n=3 \& 3$ per treatment group).

B. Quantification of Ucp-1 gene expression for 24hrs treatment with $10 \mathrm{mM}$ Dipropyl Malonate (DPM) (Chemical structure shown above). ( $n=3 \& 3$ per treatment group).

C. Quantification of $U c p-1$ gene expression for $24 \mathrm{hrs}$ treatment with $10 \mathrm{mM}$ Dimethyl malonate (DMM) (Chemical structure shown above). ( $n=3 \& 3$ per treatment group).

D. Quantification of Ucp-1 gene expression for $24 \mathrm{hrs}$ treatment with $10 \mathrm{mM}$ Dibenzyl Malonate (DBnM) (Chemical structure shown above). ( $n=3 \& 3$ per treatment group).

E. Quantification of Ucp-1 gene expression for $24 \mathrm{hrs}$ treatment with $10 \mathrm{mM}$ meldrum's acid (MEL) (Chemical structure shown above). ( $n=3 \& 3$ per treatment group). significant using an unpaired two sided student t-test and denoted as *. 


\section{References}

583 1. van Marken Lichtenbelt, W. D. et al. Cold-Activated Brown Adipose Tissue in Healthy Men. N. Engl. J. Med. 360, 1500-1508 (2009).

585

586

587

588

589

590

591

592

593

594

595

596

597

598

599

600

601

602

603

604

605

2. Virtanen, K. A. et al. Functional Brown Adipose Tissue in Healthy Adults. N. Engl. J. Med. 360, 1518-1525 (2009).

3. Cypess, A. M. et al. Identification and Importance of Brown Adipose Tissue in Adult Humans. N. Engl. J. Med. 360, 1509-1517 (2009).

4. Kajimura, S. \& Saito, M. A New Era in Brown Adipose Tissue Biology: Molecular Control of Brown Fat Development and Energy Homeostasis. Annu. Rev. Physiol. 76, 225249 (2014).

5. Wu, J., Cohen, P. \& Spiegelman, B. M. Adaptive thermogenesis in adipocytes: Is beige the new brown? Genes Dev. 27, 234-250 (2013).

6. Yoneshiro, T. et al. Recruited brown adipose tissue as an antiobesity agent in humans. J. Clin. Invest. 123, 3404-3408 (2013).

7. Hanssen, M. J. W. et al. Short-term cold acclimation improves insulin sensitivity in patients with type 2 diabetes mellitus. Nat. Med. 21, 863-865 (2015).

8. Bartelt, A. et al. Brown adipose tissue activity controls triglyceride clearance. Nat. Med. 17, 200-205 (2011).

9. Stanford, K. I. et al. Brown adipose tissue regulates glucose homeostasis and insulin sensitivity. J. Clin. Invest. 123, 215-223 (2013).

10. Arch, J. R. S. Challenges in $\beta 3$-adrenoceptor agonist drug development. Ther. Adv. Endocrinol. Metab. 2, 59-64 (2011).

11. Kivelä, R. et al. Endothelial Cells Regulate Physiological Cardiomyocyte Growth via VEGFR2-Mediated Paracrine Signaling. Circulation 139, 2570-2584 (2019).

606 12. Noireaud, J. \& Andriantsitohaina, R. Recent Insights in the Paracrine Modulation of

607 Cardiomyocyte Contractility by Cardiac Endothelial Cells. Biomed Res. Int. 2014, 1-10

608 (2014).

609 13. Wei, H. et al. Characterization of the polarized endothelial secretome. FASEB J. 33,

610 12277-12287 (2019).

611 14. Haywood, N. J. et al. Endothelial IGF-1 receptor mediates crosstalk with the gut wall

612

613

614

615 to regulate microbiota in obesity. EMBO Rep. 22, (2021).

15. Swinburn, B. A. et al. The global obesity pandemic: shaped by global drivers and local environments. Lancet 378, 804-814 (2011).

16. Mozaffarian, D., Hao, T., Rimm, E. B., Willett, W. C. \& Hu, F. B. Changes in Diet and

616

617 Lifestyle and Long-Term Weight Gain in Women and Men. N. Engl. J. Med. 364, 2392-2404 (2011).

618 17. Schoeller, D. A. The energy balance equation: looking back and looking forward are

619 two very different views. Nutr. Rev. 67, 249-254 (2009).

620 18. GBD. Health Effects of Overweight and Obesity in 195 Countries over 25 Years. N.

621 Engl. J. Med. 377, 13-27 (2017).

622 19. Hruby, A. et al. Determinants and Consequences of Obesity. Am. J. Public Health

623 106, 1656-1662 (2016).

624 20. Pellegrinelli, V., Carobbio, S. \& Vidal-Puig, A. Adipose tissue plasticity: how fat depots respond differently to pathophysiological cues. Diabetologia 59, 1075-1088 (2016). 21. Kajimura, S., Spiegelman, B. M. \& Seale, P. Brown and Beige Fat: Physiological Roles beyond Heat Generation. Cell Metab. 22, 546-559 (2015).

22. Haywood, N. J., Slater, T. A., Matthews, C. J. \& Wheatcroft, S. B. The insulin like growth factor and binding protein family: Novel therapeutic targets in obesity \&amp; diabetes. Mol. Metab. 19, (2019).

631 23. Imrie, H. et al. Vascular insulin-like growth factor-I resistance and diet-induced

632 obesity. Endocrinology 150, 4575-4582 (2009).

633 24. Cubbon, R. M., Kearney, M. T. \& Wheatcroft, S. B. Endothelial IGF-1 Receptor

634 Signalling in Diabetes and Insulin Resistance. Trends Endocrinol. Metab. 27, 96-104 (2016). 
25. Gogg, S., Nerstedt, A., Boren, J. \& Smith, U. Human adipose tissue microvascular endothelial cells secrete PPARy ligands and regulate adipose tissue lipid uptake. JCI Insight 4, (2019).

26. Hammel, J. H. \& Bellas, E. Endothelial cell crosstalk improves browning but hinders white adipocyte maturation in 3D engineered adipose tissue. Integr. Biol. 12, 81-89 (2020). 27. Crewe, C. et al. An Endothelial-to-Adipocyte Extracellular Vesicle Axis Governed by Metabolic State. Cell 175, 695-708.e13 (2018).

28. Kershaw, E. E. \& Flier, J. S. Adipose tissue as an endocrine organ. J. Clin. Endocrinol. Metab. 89, 2548-2556 (2004).

29. Kusminski, C. M., Bickel, P. E. \& Scherer, P. E. Targeting adipose tissue in the treatment of obesity-associated diabetes. Nat. Rev. Drug Discov. 15, 639-660 (2016). 30. Tran, K.-V. et al. The Vascular Endothelium of the Adipose Tissue Gives Rise to Both White and Brown Fat Cells. Cell Metab. 15, 222-229 (2012).

31. Fernandez-Gomez, F. J. et al. Malonate induces cell death via mitochondrial potential collapse and delayed swelling through an ROS-dependent pathway. Br. J. Pharmacol. 144, 528-537 (2005).

32. Kelso, G. F. et al. Selective Targeting of a Redox-active Ubiquinone to Mitochondria within Cells. J. Biol. Chem. 276, 4588-4596 (2001).

33. Sharp, L. Z. et al. Human BAT Possesses Molecular Signatures That Resemble Beige/Brite Cells. PLoS One 7, e49452 (2012).

34. Fisher, f. M. et al. FGF21 regulates PGC-1 and browning of white adipose tissues in adaptive thermogenesis. Genes Dev. 26, 271-281 (2012).

35. Hondares, E. et al. Thermogenic Activation Induces FGF21 Expression and Release in Brown Adipose Tissue. J. Biol. Chem. 286, 12983-12990 (2011).

36. Flippo, K. H. \& Potthoff, M. J. Metabolic Messengers: FGF21. Nat. Metab. 3, 309317 (2021).

37. Hill, C. M. et al. Low protein-induced increases in FGF21 drive UCP1-dependent metabolic but not thermoregulatory endpoints. Sci. Rep. 7, 8209 (2017).

38. Kwon, M. M., O'Dwyer, S. M., Baker, R. K., Covey, S. D. \& Kieffer, T. J. FGF21Mediated Improvements in Glucose Clearance Require Uncoupling Protein 1. Cell Rep. 13, 1521-1527 (2015).

39. Prag, H. A. et al. Ester Prodrugs of Malonate with Enhanced Intracellular Delivery Protect Against Cardiac Ischemia-Reperfusion Injury In Vivo. Cardiovasc. Drugs Ther. (2020) doi:10.1007/s10557-020-07033-6.

40. Baumann, C. \& Stare, F. The effect of malonate on tissue respiration. J Biol Chem 133, 183-191 (1940).

41. Mills, E. L. et al. Accumulation of succinate controls activation of adipose tissue thermogenesis. Nature 560, 102-106 (2018).

42. Dietrich, P., Dragatsis, I., Xuan, S., Zeitlin, S. \& Efstratiadis, A. Conditional mutagenesis in mice with heat shock promoter-driven cre transgenes. Mamm. Genome 11, 196-205 (2000).

43. Muzumdar, M. D., Tasic, B., Miyamichi, K., Li, L. \& Luo, L. A global doublefluorescent Cre reporter mouse. genesis 45, 593-605 (2007).

44. Stubbins, R. E., Holcomb, V. B., Hong, J. \& Núñez, N. P. Estrogen modulates abdominal adiposity and protects female mice from obesity and impaired glucose tolerance. Eur. J. Nutr. 51, 861-870 (2012).

45. Griffin, C., Lanzetta, N., Eter, L. \& Singer, K. Sexually dimorphic myeloid inflammatory and metabolic responses to diet-induced obesity. Am. J. Physiol. Integr. Comp. Physiol. 311, R211-R216 (2016).

46. Haywood, N. J. et al. Insulin-Like Growth Factor Binding Protein 1 Could Improve Glucose Regulation and Insulin Sensitivity Through Its RGD Domain. Diabetes 66, 287-299 (2017).

47. Abbas, A. et al. The insulin-like growth factor-1 receptor is a negative regulator of nitric oxide bioavailability and insulin sensitivity in the endothelium. Diabetes 60, 2169-2178 (2011). 
690 48. Watt, N. T. et al. Endothelial SHIP2 Suppresses Nox2 NADPH Oxidase-Dependent

691 Vascular Oxidative Stress, Endothelial Dysfunction, and Systemic Insulin Resistance.

692 Diabetes 66, 2808-2821 (2017).

693 49. Whitehead, A. et al. Brown and beige adipose tissue regulate systemic metabolism

694 through a metabolite interorgan signaling axis. Nat. Commun. 12, 1905 (2021).

695 50. Roberts, L. D. et al. $\beta$-Aminoisobutyric Acid Induces Browning of White Fat and

696 Hepatic $\beta$-oxidation and is Inversely Correlated with Cardiometabolic Risk Factors. Cell

697 Metab. 19, 96-108 (2014).

698 51. Liang, W., Menke, A. L., Driessen, A., Koek, G. H. \& Lindeman, J. H. Establishment

699 of a General NAFLD Scoring System for Rodent Models and Comparison to Human Liver

700 Pathology. PLoS One 9, 1-17 (2014).

701 52. Greenway, F. L. et al. An Assay to Measure Angiogenesis in Human Fat Tissue.

702 Obes. Surg. 17, 510-515 (2007).

703 53. Mohammadi, M. et al. Crystal structure of an angiogenesis inhibitor bound to the FGF receptor tyrosine kinase domain. EMBO J. 17, 5896-5904 (1998).

\section{Methods}

708 Mice with tamoxifen-inducible endothelial cell specific knockdown of the IGF-1R receptor 709 (ECIGF-1R ${ }^{\mathrm{KD}}$ ) and their lox/lox control littermates, were bred in house from founder animals

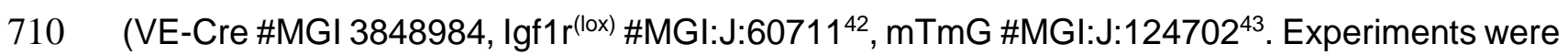
711 carried out under the authority of UK Home Office Licence P144DD0D6. Mice were group 712 housed in cages of up to five animals. Only male mice were used for experimental procedures

713 to prevent variability associated with the estrous cycle on adiposity and metabolic readouts 71444,45 . Cages were maintained in humidity- and temperature-controlled conditions (humidity $71555 \%$ at $22^{\circ} \mathrm{C}$ ) with a $12 \mathrm{hr}$ light-dark cycle. Genotyping was carried out by Transnetyx 716 commercial genotyping using ear biopsies'. At 5 weeks old, mice were injected with tamoxifen 717 (T5648 Sigma, dissolved in, Corn Oil - also from Sigma, C8267) (1mg/day intra-peritoneal for 7185 consecutive days). To induce obesity, 8 week old male mice received high fat diet ad libitum 719 for either 2 weeks or 8 weeks (60\% of energy from fat) (F1850, Bioserve) with the following composition: protein $20.5 \%$, fat $36 \%$ and carbohydrate $36.2 \%(5.51 \mathrm{kcal} / \mathrm{g})$.

Insulin and glucose tolerance testing

723 Mice were fasted overnight prior to glucose tolerance tests or for $2 \mathrm{hr}$ prior to insulin tolerance

724 tests. Blood glucose was measured using a handheld Glucose Meter (Accu-Chek Aviva). An 725 intra-peritoneal injection of glucose $(1 \mathrm{mg} / \mathrm{g})$ or recombinant human insulin (Actrapid; Novo 726 Nordisk) $(0.75 \mathrm{IU} / \mathrm{kg})$ was given and glucose concentration measured at $30 \mathrm{~min}$ intervals for $7272 \mathrm{hrs}$ from the point of glucose/insulin administration. Mice were not restrained between measurements ${ }^{46}$. Data were analysed using GraphPad Prism Area under the curve (AUC) calculations. 
731 Genotyping of endothelial cell specific knockdown of the IGF-1R receptor (ECIGF-1R $R^{K D}$ ) and 732 their lox/lox control littermates

733 VE-cre reaction mix; $0.5 \mu \mathrm{l} 10 \mu \mathrm{M}$ Forward Primer: 5'-

734 GCATtACCGgtcGATGCAACGAGTGATGAG $\quad-3$ ' $0.5 \mu \mathrm{l} \quad 10 \mu \mathrm{M}$ Reverse Primer: 5'735 GAGTGAACGAACCTGGTCGAAATCAGTGCG -3' 10 $\mu \mathrm{l}$ x2 Bio mix red PCR Master Mix, 13 $\mu \mathrm{l}$ 736 water and $1 \mu$ l extracted DNA. PCR cycle as follows; Initial denaturation $95^{\circ} \mathrm{C}$ for $1 \mathrm{~min}$, 737 denaturation $95^{\circ} \mathrm{C}$ for $15 \mathrm{sec}$, annealing $51^{\circ} \mathrm{C}$ for $30 \mathrm{sec}$, extension $72^{\circ} \mathrm{C}$ for 1 min and final 738 extension $72^{\circ} \mathrm{C}$ for 6 min. Denaturation, annealing and extension repeated for 35 cycles. PCR 739 products were then run on a $1.5 \%$ agarose gel for $1 \mathrm{hr}$ at $110 \mathrm{~V}$, with a $100 \mathrm{bp}$ ladder. Expected 740 product sizes are Cre Positive - 408 bp.

742 IGF-1R lox reaction mix; $1 \mu \mathrm{l}$ 10 $\mu \mathrm{M}$ Forward Primer1: 5'-CTTCCCAGCTTGCTACTCTAG G -

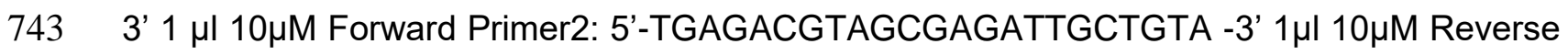
744 Primer: 5'-CAGGCTTGCAATGAGACATGGG -3' 10 $\mu \mathrm{l}$ x2 Bio mix red PCR MasterMix, $11 \mu \mathrm{l}$ 745 water and $1 \mu$ lextracted DNA. PCR cycle as follows; Initial denaturation $94^{\circ} \mathrm{C}$ for $4 \mathrm{~min}$, 746 denaturation $94^{\circ} \mathrm{C}$ for $45 \mathrm{sec}$, annealing $61^{\circ} \mathrm{C}$ for $45 \mathrm{sec}$, extension $72^{\circ} \mathrm{C}$ for 1 min and final 747 extension $72^{\circ} \mathrm{C}$ for $5 \mathrm{~min}$. Denaturation, annealing and extension repeated for 35 cycles. PCR 748 products were then run on a $1.5 \%$ agarose gel for $1 \mathrm{hr}$ at $110 \mathrm{~V}$, with a $100 \mathrm{bp}$ ladder. Expected 749 products sizes are; Wild type -120 bp, Homozygous - 220 bp and Heterozygous - 120 \& 220 750 bp.

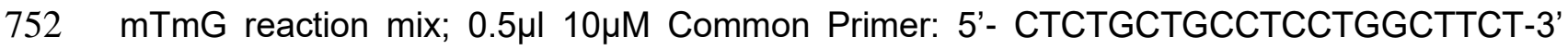

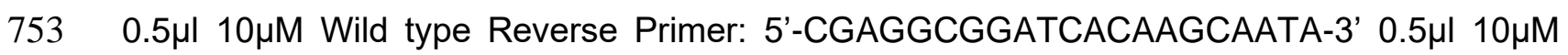
754 Mutant Reverse Primer: 5'-TCAATGGGCGGGGGTCGTT-3' 10 $\mu \mathrm{l}$ x2 Bio mix red PCR Master Mix, $12.5 \mu \mathrm{l}$ water and $1 \mu \mathrm{l}$ extracted DNA. PCR cycle as follows; Initial denaturation $94^{\circ} \mathrm{C}$ for $2 \mathrm{~min}$, denaturation $94^{\circ} \mathrm{C}$ for $30 \mathrm{sec}$, annealing $62^{\circ} \mathrm{C}$ for $30 \mathrm{sec}$, extension $72^{\circ} \mathrm{C}$ for $30 \mathrm{sec}$ and final extension $72^{\circ} \mathrm{C}$ for $10 \mathrm{~min}$. Denaturation, annealing and extension repeated for 35 cycles. PCR products were then run on a $1.5 \%$ agarose gel for $1 \mathrm{hr}$ at $110 \mathrm{~V}$, with a $100 \mathrm{bp}$ ladder. Expected product sizes are; Wild type - 330 bp, Homozygous - 250 bp, and Heterozygous - $250 \& 350 \mathrm{bp}$.

Confirmation of tamoxifen induction of $m T$ to $m G$

763 Founder mTmG mice were obtained from the Jackson Laboratory (Bar Harbor, ME, USA). In the absence of Cre recombinase, mTmG mice constitutively express mTdTomato, a nonoligomerizing DsRed variant. After tamoxifen induction and therefore following exposure to Cre recombinase and excision of the mTdTomato expression cassette, the rearranged $\mathrm{mTmG}$ transgene converts to the expression of mGFP (green fluorescent protein). Both mTdTomato 
and mGFP are membrane-targeted, allowing for delineation of single cells using fluorescence microscopy. Mice were perfuse-fixed with 4\% paraformaldehyde (PFA). Femoral arteries were excised, permeabilised (0.1\% TritonX-100 in PBS) and blocked (Serum free protein block, DAKO), before overnight incubation with a rabbit polyclonal antibody to mouse CD31 (ab28364, Abcam) followed by overnight incubation with a goat polyclonal anti-rabbit conjugated to Chromeo642 (ab60319, Abcam, UK). Arteries were then mounted en face on slides using DAPI (DAPI-Fluoromount-G, Southern Biotech) to define nuclei. Confocal microscopy (LSM 700, Zeiss, UK) was used to define CD31, mGFP and mTdTomato fluorescence.

\section{Primary endothelial cell isolation and culture}

779 Primary endothelial cells (PECs) were isolated from lungs, as previously reported ${ }^{47,48}$. Briefly, 780 lungs were harvested, washed, finely minced, and digested in Hanks' balanced salt solution containing 0.18 units $/ \mathrm{mL}$ collagenase $\left(10 \mathrm{mg} / \mathrm{mL}\right.$; Roche) for $45 \mathrm{~min}$ at $37^{\circ} \mathrm{C}$. The digested tissue was filtered through a 70- $\mu \mathrm{m}$ cell strainer and centrifuged at 1,000 RPM for $10 \mathrm{~min}$. The cell pellet was washed with PBS/0.5\% BSA, centrifuged, re-suspended in $1 \mathrm{~mL} P B S / 0.5 \%$ BSA, and incubated with $1 \times 10^{6}$ CD146 antibody-coated beads (Miltenyi Biotech, 130-092007 ) at $4^{\circ} \mathrm{C}$ for $30 \mathrm{~min}$. Bead-bound PEC were separated from non-bead-bound cells using a magnet. Cells were re-suspended in $2 \mathrm{ml}$ supplemented endothelial growth medium-MV2 (PromoCell) and seeded on a 6 well fibronectin coated plates. Cells were cultured at $37^{\circ} \mathrm{C}$ in $5 \% \mathrm{CO}_{2}$ with twice-weekly media changes until confluent.

\section{Quantification of protein expression}

791 Cells were lysed or tissue mechanically homogenised in lysis buffer (Extraction buffer, 792 FNN0011) and protein content was quantified by a BCA assay (Sigma-Aldrich, St. Louis, MO).

793 Twenty micrograms of protein were resolved on a 4-12\% Bis-Tris gel (Bio-Rad, Hertfordshire, 794 UK) and transferred to nitrocellulose membranes. Membranes were probed with antibodies 795 diluted in 5\% BSA (IGF-1R and IR, Cell signalling \#9750 and \#3025 respectively), before incubation with appropriate secondary horseradish peroxidase-conjugated antibody. Blots were visualised with Immobilon Western Chemiluminescence HRP Substrate (Merck Millipore,

798 Hertfordshire, UK) and imaged with Syngene chemiluminescence imaging system (SynGene,

799 Cambridge, UK). Densitometry was performed in ImageJ.

\section{Plasma samples}

802 Fasting plasma samples were collected from the lateral saphenous vein (EDTA collection 803 tubes Sarstedt 16.444) and spun at 10,000 RPM for 10min in a bench top centrifuge. Fasting 804 plasma insulin (90080, CrystalChem), IGF-I (MG100, R and D systems), leptin (EZML-82K, 
Merk-Millipore), adiponectin (EZMADP-60K, Merk-Millipore) triglycerides (Abcam Ab65336) and free fatty acids (Abcam, ab65341) were measured as per manufactures instructions.

\section{Metabolic phenotyping}

809 Metabolic parameters were measured by indirect calorimetry using Comprehensive Lab

810 Animal Monitoring Systems (CLAMS)(Columbus Instruments). In brief, mice were individually

811 housed for 5 days and measurement of their oxygen consumption, carbon dioxide production, 812 food intake, and locomotor activity were continuously recorded ${ }^{49}$. For each mouse, a full 24

813 hour period, taking into account sleep and wake cycles, was analysed after an acclimatisation

814 period ${ }^{50}$. Core body temperature was measured using a rectal temperature probe (Vevo2100

815 (Visualsonics, FujuFilm) with an Indus rectal temperature probe).

816

\section{Murine tissue samples}

818 After either 2 or 8 weeks of high fat feeding, all mice were sacrificed using terminal anaesthesia and organ weights measured using a standard laboratory balance.

\section{Quantification of gene expression}

822 RNA was isolated from tissue and cells samples (NEB, T2010S). The concentration of RNA 823 in each sample ( $\mathrm{ng} / \mathrm{ul}$ ) was measured using a Nanodrop. cDNA was reverse transcribed from 824 the RNA samples (NEB, E3010L). Quantitative PCR (qPCR) was performed using a Roche 825 LightCycler 480 Instrument II, using SYBR Green PCR Master Mix (Bio-Rad, 1725120) and 826 relevant primers (Table 1). The 'cycles to threshold' (cT) was measured for each well, the 827 average of triplicate readings for each sample taken, normalised to GAPDH, and finally the differential expression of each gene was calculated for each sample.

830 Histological assessment of adipocyte size, fibrous tissue and non-alcoholic fatty liver disease

831 Samples for histology were fixed in 4\% PFA for at least 24hrs and then processed into paraffin 832 blocks. $5 \mu \mathrm{m}$ sections were taken, slides were stained with haematoxylin and eosin to assess 833 gross morphology or Picro-sirius red for collagen deposition. Slides were imaged using an 834 Olympus BX41 microscope at 10x and 20x magnification. For assessment of adipocyte size, 835 three separate fields of view for each sample were assessed. For each one, the average was 836 taken of 20 randomly selected independent cells measured using ImageJ. For collagen 837 deposition, the percentage of the sample staining positive for collagen was measured using 838 thresholding in ImageJ, and again was taken as the average in at least three independent 839 areas of the sample. For assessment of non-alcoholic fatty liver disease (NAFLD) in sections 840 of murine liver, a validated rodent NAFLD scoring system was used ${ }^{51}$, which takes into account 841 micro and macro-steatosis, inflammation and hypertrophy. Each sample was assessed by at 
842 least two blinded independent verifiers ( $\mathrm{NH}, \mathrm{KB}$ or $\mathrm{NW})$, and the average score per sample

843 taken.

844

\section{Quantification of tissue vascularity}

846 Adipose tissue was fixed in 1\% paraformaldehyde (PFA), and allowed to fix for $2 \mathrm{hrs}$ at room 847 temperature; samples were transferred into phosphate buffered saline (PBS) for longer 848 storage. Samples were incubated overnight with lectin from Ulex europaeus Alexa Fluor 594 849 (73873, Sigma) (For human samples) or Isolectin B4 Alexa Fluor 647 (I32450, Thermo Fisher 850 Scientific) (for murine samples), diluted 1:100 in 5\% BSA in PBS at $4^{\circ} \mathrm{C}$. After washing with 851 PBS, they were incubated with HCS LipidTOX (H34475, Thermo Fisher Scientific) diluted $8521: 200$ in PBS for 20 mins at room temperature. Whole tissue sections were then mounted onto 853 slides beneath cover slips using a silicone spacer (Grace bio-labs, 664113), with Prolong Gold 854 (P36930, Thermo Fisher Scientific). Vascular density (the proportion of each image stained 855 with lectin) was measured using thresholding in ImageJ. Green staining is a composite of GFP and Lipidtox in mice samples, green is not quantified.

858 Organs (Muscle and liver) were harvested under terminal anaesthesia and fixed in 4\% PFA 859 for $1 \mathrm{hr}$ at room temperature. Organs were then embedded in Optimal Cutting Temperature 860 compound (OCT) (Cellpath, KMA-0100-00A) and stored at -80 until sectioned. 10 $\mathrm{MM}$ sections 861 were taken using a Leica CM3050 S Research Cryostat. Slides were blocked and 862 permeabilised in PBS $+0.25 \%$ Triton-X100 $+1 \%$ BSA + for one hour, then stained with 863 Isolectin B4-Alexa Fluor-488 (Invitrogen I21411) at 1/100 in PBS + 0.25\% Triton + 1\% BSA

864 for $1 \mathrm{hr}$. Slides were washed three times in PBS and mounted with a coverslip using Prolong 865 Gold with DAPI (P36931, ThermoFisher). Slides were then imaged using laser scanning confocal microscopy (LSM880, Zeiss), with 8 areas of each sample imaged. Vascular density (the proportion of each image stained with IB4) was measured using thresholding in ImageJ.

\section{Assessment of neovascularisation in white adipose tissue}

870 Angiogenesis assays from adipose tissue were performed using a modified technique based 871 on previously published methods ${ }^{52}$. In sterile conditions, any surface blood vessels were 872 dissected from the adipose tissue sample, before it was cut into pieces no bigger than $1 \mathrm{~mm}^{3}$. 873 For each sample, at least 20 sections were embedded into a fibrin matrix. The fibrin matrix 874 was achieved by combining $12.5 \mathrm{ul}$ of $50 \mathrm{U} / \mathrm{ml}$ thrombin (Sigma-Aldrich T-3399) with 500ul of 875 a mix containing $4 \mathrm{U} / \mathrm{ml}$ aprotinin (Sigma-Aldrich A-1153) and $2 \mathrm{mg} / \mathrm{ml}$ fibrinogen type 1 876 (Sigma-Aldrich F-8630), and adding a piece of adipose tissue into the well before the matrix 877 had set. The plates were then incubated at room temperature for 20 minutes, and then at $37^{\circ} \mathrm{C}$ 878 for a further 20 minutes to ensure that the matrix had fully formed around the piece of adipose 
879 tissue. One millilitre of media was then carefully pipetted onto the top of each well, and plates

880 were cultured at $37^{\circ} \mathrm{C}, 5 \% \mathrm{CO}_{2}$ for up to 7 days. The media was discarded and replaced every

881 other day throughout the culture period. Each day, the samples were imaged at $4 x$

882 magnification on Olympus florescent microscope CKX41 and number of endothelial sprouts

883 coming from each piece of fat was counted. For each sample, the average number of sprouts

884 per section was calculated, as well as the number of sections which had sprouted.

Human adipose tissue explants

887 Human subcutaneous white adipose tissue was obtained after informed consent from patients 888 undergoing pacemaker implantation at Leeds Teaching Hospitals NHS Trust, Leeds, United

889 Kingdom, after ethical approval (REC: 11/YH/0291). Adipose tissue was removed from the 890 area between the skin and pectoralis major, under local anaesthetic (1\% lidocaine). Patient 891 demographics are provided in table 2.

892

893 Conditioning media

894 When PECS reached confluency, supplemented growth media was removed and replaced with basal endothelial growth medium-MV2 (Promocell) for 24hrs. Conditioned media was then removed and used in further experiments as described.

Quantification of browning in human adipocytes

899 Human primary adipocytes (PromoCell, C-12730) were seeded $\left(10,000 \mathrm{cells} / \mathrm{cm}^{2}\right)$ in 24 well 900 plates (Costar, Corning, NY, USA) and grown until confluence $\left(37^{\circ} \mathrm{C}, 5 \% \mathrm{CO}_{2}\right)$ in PromoCell 901 Preadipocyte Growth Medium (C-27410, $0.05 \mathrm{~mL} / \mathrm{mL}$ fetal calf serum, $0.004 \mathrm{~mL} / \mathrm{mL}$ 902 endothelial cell growth supplement, $10 \mathrm{ng} / \mathrm{mL}$ epidermal growth factor, $1 \mu \mathrm{g} / \mathrm{mL}$ 903 hydrocortisone, $90 \mu \mathrm{g} / \mathrm{mL}$ heparin) as previously described ${ }^{49}$. To differentiate confluent pre904 adipocytes, growth medium was replaced by PromoCell Adipocyte Differentiation Medium (C905 27436, $8 \mu \mathrm{g} / \mathrm{mL}$ d-Biotin, $0.5 \mu \mathrm{g} / \mathrm{mL}$ insulin, $400 \mathrm{ng} / \mathrm{mL}$ dexamethasone, $44 \mu \mathrm{g} / \mathrm{mL}$ IBMX, 9 $906 \mathrm{ng} / \mathrm{mL}$ L-thyroxine, $3 \mu \mathrm{g} / \mathrm{ml}$ ciglitazone) for 48 hours (day 0 ). Differentiation medium was 907 subsequently replaced (day 2) with PromoCell Adipocyte Nutrition Medium (C-27438, 0.03 $908 \mathrm{~mL} / \mathrm{mL}$ fetal calf serum, $8 \mu \mathrm{g} / \mathrm{mL}$ d-Biotin, $0.5 \mu \mathrm{g} / \mathrm{mL}$ insulin, $400 \mathrm{ng} / \mathrm{mL}$ dexamethasone) for 909 the remainder of the differentiation period (up to day 14). All Cell medium was supplemented 910 with $1 \%$ penicillin-streptomycin (10,000 units/mL penicillin, $10 \mathrm{mg} / \mathrm{mL}$ streptomycin). 911 Conditioned media was then added to the differentiated human adipocytes for 24hrs before 912 the cells were lysed and RNA extracted for gene expression analysis. To determine if browning 913 was caused by a protein or small molecule, parallel experiments were conducted whereby the 914 conditioned media was boiled for 5 mins at $95^{\circ} \mathrm{C}$, to denature any proteins, before being added 915 to the human adipocytes. 
Metabolite \& pro-drug screening

918 Mouse 3T3-L1 preadipocytes were cultured in 10\% (v/v) CS/DMEM containing $4.5 \mathrm{~g} / \mathrm{l}$ glucose

919 and 1mM Sodium Pyruvate and supplemented with 1XAntibiotic Antimycotic Solution and

920 incubated at $37^{\circ} \mathrm{C}$ in $5 \% \mathrm{CO}_{2}$ for two days upon splitting. Two days after splitting, the media

921 was replaced by $10 \%$ (v/v) FBS/DMEM to grow the cells to confluency. After two days of post-

922 confluency (equivalence of day 0 ), adipocyte differentiation was initiated with MDI induction

923 media (10\% FBS/DMEM, 0.5mM IBMX, $1 \mu \mathrm{M}$ dexamethasone and $1 \mu \mathrm{g} / \mathrm{mL}$ insulin). On day 2 ,

924 the MDI induction media was replaced by insulin media (10\% FBS/DMEM supplemented with

$9251 \mu \mathrm{g} / \mathrm{mL}$ insulin). From day 4 onwards, the media was replaced by $10 \%$ FBS/DMEM every two

926 days. Full differentiation was achieved between day 7 and day 10. Mature adipocytes were

927 subjected to metabolite stimulation. Metabolites, Malonic acid ${ }^{31}$ or pro-drugs (Table 3) and

928 their solvents, DPBS or DMSO, were applied for 24 hours at $37^{\circ} \mathrm{C}$ in $5 \% \mathrm{CO}_{2}$.

929

930 Adiponectin secretion

931 After stimulation of the mature 3T3-L1 adipocytes, the conditioned media was collected and

932 centrifuged at $13,400 \mathrm{rpm}$ for $10 \mathrm{~min}$ at $4^{\circ} \mathrm{C}$ to pellet cell debris. The supernatants were then

933 used to quantify the level of adiponectin using Mouse Adiponectin ELISA kit (Merck Millipore

934 \#EZMADP-60K) according to the manufacturer's instructions.

935

936

FGFR1 blocker

937 20nM PD173074 (Apexbio \#A8253) was applied an hour prior to 24-hour malonic acid 938 treatment ${ }^{53}$.

939

Mitochondria-targeted antioxidant

941 100nM Mitoquinone (MitoQ; MedChemExpress LLC \#HY-100116A) was applied 30min prior to 24-hour malonic acid treatment ${ }^{41}$.

Separation of conditioned media into aqueous and lipid fractions

$945600 \mu \mathrm{l}$ of 2:1 methanol:chloroform was added to $1 \mathrm{ml}$ of conditioned media, followed by $200 \mu \mathrm{l}$

946 of water and an additional $200 \mu \mathrm{l}$ of chloroform, vortexed and then centrifuged at $13.1 \mathrm{~g}$ for

947 20mins. The top layer (aqueous layer containing the aqueous metabolites) was pipetted off 948 and placed into the evacuation centrifuge at $40^{\circ} \mathrm{C}$ for 6 hours. The protein disc (middle layer) 949 was discarded and the final bottom layer (containing lipid metabolites) was transferred into a 950 clean Eppendorf and left overnight in a fume hood at room temperature until all chloroform 951 had evaporated. Both the lipid and aqueous metabolites were stored at $-80^{\circ} \mathrm{C}$. 


\section{Aqueous Sample Preparation}

Samples were reconstituted in $1 \mathrm{ml}$ sample resuspension buffer (95\% acetonitrile and $5 \%$ mobile phase A). Mobile phase $A=95 \%$ water, $5 \%$ acetonitrile, $20 \mathrm{mM}$ ammonium acetate and $20 \mathrm{mM}$ ammonium hydroxide, $\mathrm{pH}=9$. Samples were vortex mixed and the extracted metabolites were transferred to a $2 \mathrm{~mL}$ glass vial.

\section{Liquid Chromatography}

A SCIEX ExionLC ${ }^{\text {TM }}$ AD HPLC system with a Luna $3 \mu \mathrm{m}$ NH2 $100 \AA$, $150 \times 4.6 \mathrm{~mm}$ column (Phenomenex) was used. Mobile phase $A=95 \%$ water, $5 \%$ acetonitrile, $20 \mathrm{mM}$ ammonium acetate and $20 \mathrm{mM}$ ammonium hydroxide, $\mathrm{pH}=9$; Mobile phase $\mathrm{B}=95 \%$ acetonitrile and $5 \%$ mobile phase $A$ and $20 \mathrm{mM}$ ammonium hydroxide. The flow rate was set at $350 \mu \mathrm{L} / \mathrm{min}$. The wash solvent for the autosampler was 20/20/60 methanol/acetonitrile/isopropanol. The injection volume was $2 \mu \mathrm{L}$, and the column was kept at $40^{\circ} \mathrm{C}$. The gradient method was $100 \%$ B for 2 minutes, then to $85 \%$ B for 3 minutes, then to $30 \%$ for $10 \mathrm{~min}$, then to $2 \%$ B for $5 \mathrm{~min}$, then $100 \%$ for $10 \mathrm{~min}$.

\section{Mass Spectrometry}

A SCIEX QTRAP ${ }^{\circ} 6500$ + with lonDrive Turbo V source was used. MS source parameters are Curtain Gas was 30 for both (+) and (-). Collision Gas was high for both (+) and (-), lonspray voltage was 5500 for (+) and -4500 for (-). Temperature was 500 for both (+) and (), Ion source gas 1 was 35 for both (+) and (-), ion source gas was 45 for both $(+)$ and (-), delustering potential was 93 for $(+)$ and -93 for $(-)$, entrance potential was 10 for $(+)$ and -10 for $(-)$ and collision cell exit potential was 10 for $(+)$ and -10 for $(-)$.

\section{Quantification and statistical analysis}

Priori sample size calculations for animal experiments were performed using our published pilot data using the online software package from Vanderbilt University for multiple types of power analysis (https://biostat.app.vumc.org/wiki/Main/PowerSampleSize). All data are shown as mean \pm SEM. Individual mice or replicates are shown as individual data points. All image analysis was performed in ImageJ. Pearsons' correlation coefficients $(r)$ were calculated to assess the link and the degree of relation between BMI and $\mathrm{HbA1C}$ and various fat markers. Student 2-tailed unpaired t-test or one-way ANOVA (where appropriate) were used for statistical analyses and were performed with GraphPad Prism software version 7. * denotes $P \leq 0.05$ and ${ }^{* *} P \leq 0.01$. Exact details can be found in figure legends.

\section{Data availability}


989 The data that support the findings of this study are available from the corresponding author

990 upon reasonable request.

\section{Method references}

993 42. Dietrich, P., Dragatsis, I., Xuan, S., Zeitlin, S. \& Efstratiadis, A. Conditional mutagenesis in mice with heat shock promoter-driven cre transgenes. Mamm. Genome 11, 196-205 (2000).

996 43. Muzumdar, M. D., Tasic, B., Miyamichi, K., Li, L. \& Luo, L. A global double-

997 fluorescent Cre reporter mouse. genesis 45, 593-605 (2007).

998 44. Stubbins, R. E., Holcomb, V. B., Hong, J. \& Núñez, N. P. Estrogen modulates

999 abdominal adiposity and protects female mice from obesity and impaired glucose tolerance.

1000 Eur. J. Nutr. 51, 861-870 (2012).

1001 45. Griffin, C., Lanzetta, N., Eter, L. \& Singer, K. Sexually dimorphic myeloid

1002 inflammatory and metabolic responses to diet-induced obesity. Am. J. Physiol. Integr. Comp.

1003 Physiol. 311, R211-R216 (2016).

1004

46. Haywood, N. J. et al. Insulin-Like Growth Factor Binding Protein 1 Could Improve Glucose Regulation and Insulin Sensitivity Through Its RGD Domain. Diabetes 66, 287-299 (2017).

47. Abbas, A. et al. The insulin-like growth factor-1 receptor is a negative regulator of nitric oxide bioavailability and insulin sensitivity in the endothelium. Diabetes 60, 2169-2178 (2011).

48. Watt, N. T. et al. Endothelial SHIP2 Suppresses Nox2 NADPH Oxidase-Dependent Vascular Oxidative Stress, Endothelial Dysfunction, and Systemic Insulin Resistance.

Diabetes 66, 2808-2821 (2017).

1013 49. Whitehead, A. et al. Brown and beige adipose tissue regulate systemic metabolism 1014 through a metabolite interorgan signaling axis. Nat. Commun. 12, 1905 (2021).

1015 50. Roberts, L. D. et al. $\beta$-Aminoisobutyric Acid Induces Browning of White Fat and Hepatic $\beta$-oxidation and is Inversely Correlated with Cardiometabolic Risk Factors. Cell Metab. 19, 96-108 (2014).

51. Liang, W., Menke, A. L., Driessen, A., Koek, G. H. \& Lindeman, J. H. Establishment of a General NAFLD Scoring System for Rodent Models and Comparison to Human Liver Pathology. PLoS One 9, 1-17 (2014).

52. Greenway, F. L. et al. An Assay to Measure Angiogenesis in Human Fat Tissue. Obes. Surg. 17, 510-515 (2007).

1024 receptor tyrosine kinase domain. EMBO J. 17, 5896-5904 (1998)

\begin{tabular}{|ll|}
\hline Gene & Bio-rad Assay code \\
\hline Adipoq & qMmuCED0045486 \\
\hline Cidea & qMmuCID0007140 \\
\hline Cd137 & qMmuCED0047964 \\
\hline Cited1 & qMmuCED0037644 \\
\hline Cox8b & qMmuCID0020689 \\
\hline Cycs & qMmuCED0001027 \\
\hline Fgf21 & qMmuCED0025797 \\
\hline Gapdh & qMmuCED0027497 \\
\hline Lep & qMmuCID0040177 \\
\hline Ppargc1a & qMmuCID0006032 \\
\hline Ppara & qMmuCED0046526 \\
\hline Ppary & qMmuCID0018821 \\
\hline Tbx1 & qMmuCID0011851 \\
\hline
\end{tabular}




\begin{tabular}{|ll|}
\hline Tmem26 & qMmuCED0061015 \\
\hline Ucp1 & qMmuCED0047500 \\
\hline Vegfa & qMmuCED0040260 \\
\hline & \\
\hline CD137 & qHsaCID0020895 \\
\hline CIDEA & qHsaCED0003559 \\
\hline CYCS & qHsaCED0046874 \\
\hline FGF21 & qHsaCIP0032896 \\
\hline GAPDH & qHsaCED0038674 \\
\hline PPARGC1a & qHsaCID0006418 \\
\hline TMEM26 & qHsaCID0009380 \\
\hline UCP-1 & qHsaCED0043275 \\
\hline
\end{tabular}

\begin{tabular}{|ll|}
\hline & Mean (土SEM) \\
\hline Male & 28 \\
\hline Female & 15 \\
\hline Diabetes & 12 \\
\hline Age $($ Years $)$ & $68(1.98)$ \\
\hline Weight $(\mathrm{Kg})$ & $86.23(3.12)$ \\
\hline Height $(\mathrm{m})$ & $1.69(0.013)$ \\
\hline BMl $\left(\mathrm{Kg} / \mathrm{m}^{2}\right)$ & $29.65(0.79)$ \\
\hline HbA1c $\left(\mathrm{mmol}^{2} / \mathrm{mol}\right)$ & $46.75(2.469)$ \\
\hline
\end{tabular}

\section{Table 2. Patient characteristics}

\begin{tabular}{|lll|l|}
\hline Metabolite/prodrug & Conc & Manufacturer & Cat \# \\
\hline 2'-deoxycytidine triphosphate (dCTP) & $10 \mathrm{mM}$ & Thermo Scientific & R0151 \\
\hline 2'-deoxyuridinemonophosphate & $2 \mathrm{mM}$ & Santa Cruz & sc-214058 \\
\hline Arginine & $2 \mathrm{mM}$ & Alfa Aesar & 11498850 \\
\hline Citric acid specific & $2 \mathrm{mM}$ & SIGMA-ALDRICH & C0759 \\
\hline Cytidine monophosphate (CMP) & $2 \mathrm{mM}$ & Fluorochem & 47062 \\
\hline Folic acid & $11.3 \mu \mathrm{M}$ & SIGMA-ALDRICH & F7876 \\
\hline Glucosamine 6P (G6P) & $2 \mathrm{mM}$ & Santa Cruz & sc-214809 \\
\hline Glucuronic acid & $10 \mathrm{ng} / \mathrm{ml}$ & SIGMA-ALDRICH & G5269 \\
\hline Glutamine & $2 \mathrm{mM}$ & Thermo Fisher & 25030032 \\
\hline Guanosine diphosphate (GDP) & $10 \mu \mathrm{M}$ & MedChemExpress & HY113066A \\
\hline Guanosine-3'5-'cyclic monophosphate & $200 \mu \mathrm{M}$ & SIGMA-ALDRICH & G7504 \\
\hline Inosine & $100 \mu \mathrm{M}$ & Alfa Aesar & A14459.06 \\
\hline Inosine monophosphate (IMP) & $1.25 \mathrm{mM}$ & SIGMA-ALDRICH & 57510 \\
\hline Leucine & $2 \mathrm{mM}$ & G BIOSCIENCES & RC-064 \\
\hline Maleic acid & $8 \mathrm{mM}$ & Acros Organics & 10396760 \\
\hline Malonic acid & $10 \mathrm{mM}$ & Alfa Aesar & 11464523 \\
\hline NADP reduced (NADPH) & $2 \mathrm{mM}$ & Santa Cruz & sc-202725 \\
\hline Pantothenic acid & $1 \mathrm{mM}$ & SIGMA-ALDRICH & 21210 \\
\hline Phthalic acid & $10 \mu \mathrm{M}$ & SIGMA-ALDRICH & P39303 \\
\hline Proline & $15 \mathrm{nM}$ & Alfa Aesar & A10199.14 \\
\hline Quinolinic acid & $5 \mathrm{mM}$ & SIGMA-ALDRICH & 160660 \\
\hline Riboflavin & $1 \mu \mathrm{M}$ & Alfa Aesar & A11764 \\
\hline S-5-Adenosyl-L-Cysteine & $100 \mu \mathrm{M}$ & SIGMA-ALDRICH & A7772 \\
\hline & & & \\
\hline
\end{tabular}




\begin{tabular}{|llll|}
\hline Shikimic acid & $80 \mu \mathrm{M}$ & Acros Organics & 10533491 \\
\hline Thymidine diphosphate (TDP) & $2 \mathrm{mM}$ & SIGMA-ALDRICH & T9375 \\
\hline Uridine diphosphate (UDP) & $2 \mathrm{mM}$ & SIGMA-ALDRICH & U4125 \\
\hline Phosphatidylglycerol 18:0 (PG (18:0)) & $2 \mathrm{mM}$ & SIGMA-ALDRICH & $840465 \mathrm{P}$ \\
\hline alpha-linolenic acid & $300 \mu \mathrm{M}$ & Santa Cruz & sc-205545 \\
\hline Dibenzyl malonate (DBnM) & $10 \mathrm{mM}$ & Alfa Aesar & A10844.09 \\
\hline Di-tert-butyl malonate (DBM) & $10 \mathrm{mM}$ & Apollo Scientific Ltd & OR55346 \\
\hline Dimethyl malonate (DMM) & $10 \mathrm{mM}$ & Alfa Aesar & A11007.22 \\
\hline Diethyl malonate (DEM) & $10 \mathrm{mM}$ & Acros Organics & 10070760 \\
\hline Diisopropyl malonate (DPM) & $10 \mathrm{mM}$ & SIGMA-ALDRICH & 411485 \\
\hline Meldrum's acid & $10 \mathrm{mM}$ & Activate Scientific & AS33739 \\
\hline
\end{tabular}

1031 Table 3: Metabolite and prodrug details 